\title{
Diverting $T$ helper cell trafficking through increased plasticity attenuates autoimmune encephalomyelitis
}

\author{
Danielle Califano, ${ }^{1,2}$ Keith J. Sweeney, ${ }^{1}$ Hung Le, ${ }^{1,2}$ Jeffrey VanValkenburgh,,1,2 Eric Yager, ${ }^{2}$ \\ William O'Connor Jr., ${ }^{2}$ Jeffrey S. Kennedy, ${ }^{2,3}$ David M. Jones, ${ }^{4}$ and Dorina Avram ${ }^{1,2}$ \\ ${ }^{1}$ Center for Cell Biology and Cancer Research and ${ }^{2}$ Center for Immunology and Microbial Disease, Albany Medical College, Albany, New York, USA. \\ ${ }^{3}$ Division of Infectious Diseases, Wadsworth Center, New York State Department of Health, Albany, New York, USA. \\ ${ }^{4}$ Department of Pathology and Laboratory Medicine, Albany Medical Center, Albany, New York, USA.
}

\begin{abstract}
Naive $T$ helper cells differentiate into functionally distinct effector subsets that drive specialized immune responses. Recent studies indicate that some of the effector subsets have plasticity. Here, we used an EAE model and found that Th17 cells deficient in the transcription factor BCL11B upregulated the Th2-associated proteins GATA3 and IL-4 without decreasing RAR-related orphan receptor $\gamma$ (ROR $\gamma$ t), IL-17, and GM-CSF levels. Surprisingly, abnormal IL-4 production affected Th17 cell trafficking, diverting migration from the draining lymph nodes/CNS route to the mesenteric lymph nodes/gut route, which ameliorated EAE without overt colitis. T helper cell rerouting in EAE was dependent on IL-4, which enhanced retinoic acid (RA) production by dendritic cells, which further induced expression of gut-homing receptors CCR9 and $\alpha_{4} \beta_{7}$ on $B c l 11 b$ deficient $\mathrm{CD}^{+} \mathrm{T}$ cells. Furthermore, IL-4 treatment or Th2 immunization of wild-type mice with EAE caused no alteration in Th17 cytokines or ROR $\gamma \mathrm{t}$, but diverted $\mathrm{T}$ helper cell trafficking to the gut, which improved EAE outcome without overt colitis. Our data demonstrate that Th17 cells are permissive to Th2 gene expression without affecting Th17 gene expression. This Th17 plasticity has an impact on trafficking, which is a critical component of the immune response and may represent a possible avenue for treating multiple sclerosis.
\end{abstract}

\section{Introduction}

Following activation, naive $\mathrm{CD} 4^{+} \mathrm{T}$ cells differentiate into functionally distinct $\mathrm{T}$ helper subsets, each regulated by key transcription factors and producing cytokines to perform specific biological functions. Th1 cells produce IFN- $\gamma$ to control the immune response to intracellular pathogens, which is governed by the transcription factor T-bet (1), while Th2 cells are controlled by GATA3 (2) and produce IL-4, IL-5, and IL-13 during anti-parasite and allergic responses (reviewed in ref. 3 ). Th17 lineage is critical for the immune responses to extracellular bacteria and fungi (reviewed in ref. 3), produces IL-17, and is controlled by RAR-related orphan receptor $\gamma$ (ROR $\gamma \mathrm{t}$ ) (4). Th17 cells are also important in the autoimmune response in multiple sclerosis (MS) and its mouse model of EAE, with the major player being GM-CSF $(5,6)$. The recently identified $\mathrm{T}$ follicular helper (Tfh) subset produces IL-21, is controlled by Bcl6, and plays a critical role in supporting class switch recombination in B cells (reviewed in refs. 7,8 ). Th1, Th2, and Th17 subsets were once deemed highly stable, and the alternate lineage cytokines were thought to be inhibitory, such as IFN- $\gamma$ and IL-4 blocking Th17 differentiation and IL-17 production (9). However, the functional plasticity of the $T$ helper lineages has become increasingly evident, particularly during in vivo immune responses (reviewed in refs. $3,10,11)$. T helper cells were found able to express alternative lineage cytokines, such as Th17 cells expressing IFN- $\gamma(12$, 13) or Th2 cells acquiring a Th1 phenotype (14) or converting into Tfh cells (15). Tfh cells have the highest plasticity of all the

Conflict of interest: Dorina Avram is listed as an inventor in a US provisional patent application in part based on the present work.

Citation for this article: J Clin Invest. 2014;124(1):174-187. doi:10.1172/JCI70103. subsets, being able to convert to Th1, Th2, and Th17 (16). In terms of plasticity, the in vivo effects of IL-4 on Th17 immune responses remain largely undefined.

The transcription factor $B c l 11 b$ functions as both a transcriptional activator and a repressor (17-20) and has been shown to be important in thymocyte selection and survival $(21,22)$, early $\mathrm{T}$ cell commitment (23-25), and control of iNKT lineage, with a critical role in iNKT precursors as well as in glycolipid presentation by double-positive thymocytes (26). Additionally, Bcl11b controls expansion and cytotoxicity of mature $\mathrm{CD}^{+} \mathrm{T}$ cells (27) and Treg cell suppression function (28). Bcl11b is expressed in mature T helper cells; however, its role in these cells has not been described. Here we show that $B c l 11 b$ is critical for repressing the Th2 lineage program in Th17 cells. We demonstrate that Bcl11b-deficient Th17 cells expressed GATA3 and produced IL-4 and IL-5 without any impact on Ror $\gamma t$, IL-17, and GM-CSF. Unexpectedly, the major physiological consequence of elevated GATA3 and IL-4 expression was the redirection of the $\mathrm{T}$ helper cells from the draining $\mathrm{LNs}$ (dLNs), away from the CNS, to the mesenteric LNs (mLNs) and gut. We demonstrate that rerouting of the cells and EAE amelioration were dependent on abnormal IL-4 production, increased retinaldehyde dehydrogenase (RALDH) activity in dendritic cells, and retinoic acid (RA) production, which in turn caused upregulation of guthoming receptors on $\mathrm{T}$ helper cells and their diverted trafficking. Similarly, deviated $\mathrm{T}$ helper cell migration from the dLNs-CNS route to the mLNs-gut was also observed in EAE WT mice treated with IL-4 or under Th2 conditions. Thus, our results demonstrate that $B c l 11 b$ restricts GATA3 expression and IL-4 production in Th17 cells. Although GATA3 expression and IL-4 production by Th17 cells do not affect Ror $\gamma \mathrm{t}$, IL-17, and GM-CSF, they have a major impact on $\mathrm{T}$ helper cell trafficking. 
A

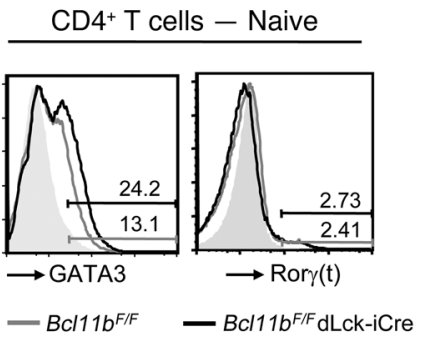

C
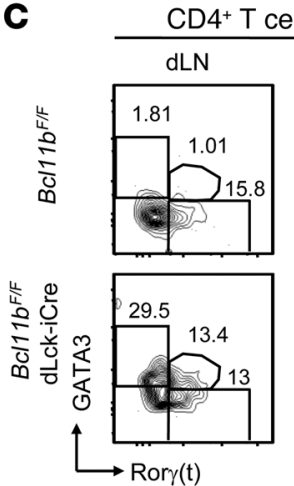

E
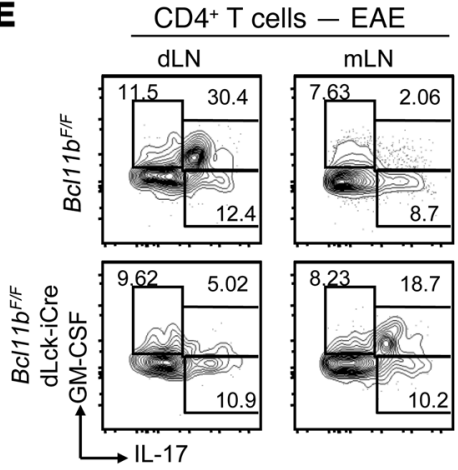

G

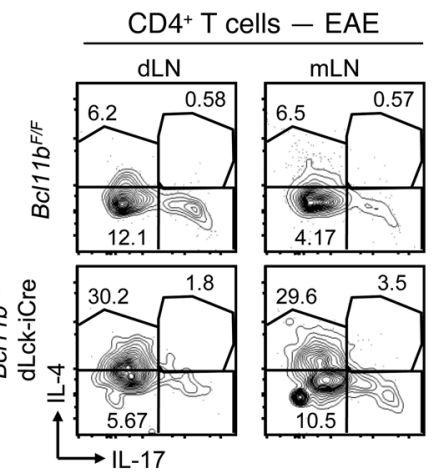

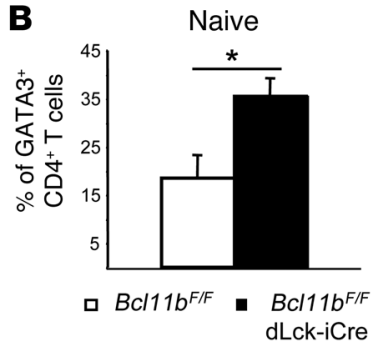

D

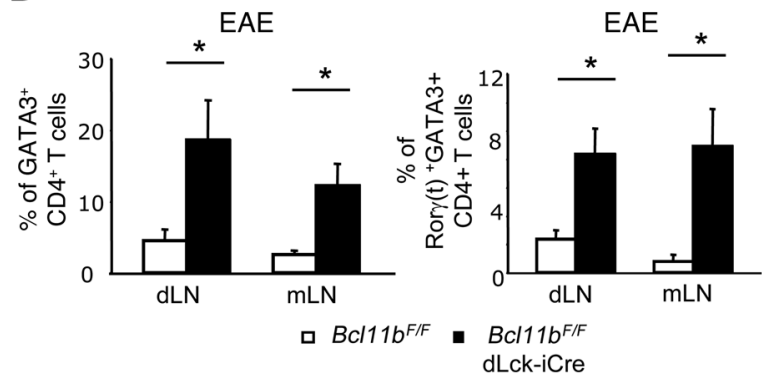

F

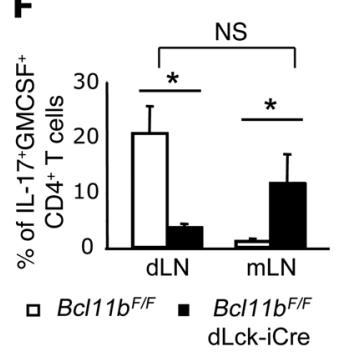

H

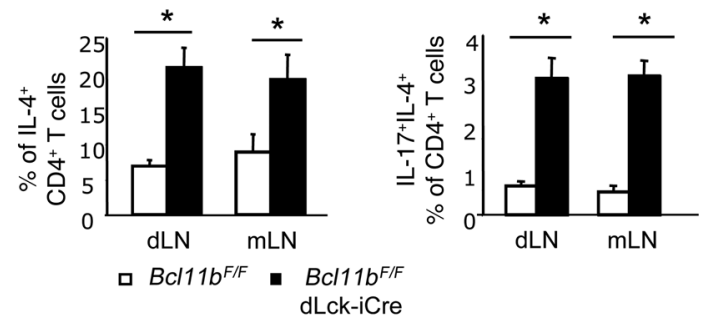

Figure 1

$\mathrm{CD}^{+}{ }^{+} \mathrm{T}$ cells of $\mathrm{Bc} / 11 \mathrm{~b}^{\mathrm{F} / \mathrm{F} /}$ dLck-iCre mice upregulate GATA3 and IL-4, but do not lose expression of Roryt, IL-17, or GM-CSF during EAE. (A) GATA3 and Roryt levels evaluated by FACS analysis in naive CD4 ${ }^{+} \mathrm{T}$ cells from $\mathrm{LNs}$ of the indicated groups of mice. Gray shaded area indicates negative control. (B) Average frequency of naive $C D 4^{+} \mathrm{T}$ cells that express GATA 3 in the indicated groups. ${ }^{*} P \leq 0.05, n=5$ (mean \pm SEM). (C) FACS analysis of GATA3 and Roryt levels in CD4+ $T$ cells in the dLNs and $\mathrm{mLNs}$ of the indicated groups on day 12 following EAE induction. (D) Average frequency of GATA3 ${ }^{+}$ (left) or GATA3 ${ }^{+}$Ror $\gamma^{+}{ }^{+}$(right) $\mathrm{CD}^{+} \mathrm{T}$ cells in the indicated groups. ${ }^{*} P \leq 0.05, n=5$ (mean \pm SEM). (E) FACS analysis of frequencies of IL-17- and GMCSF-producing $\mathrm{CD}^{+} \mathrm{T}$ cells from dLNs and mLNs of the indicated groups on day 12 after EAE induction. (F) Average frequency of IL-17+GM-CSF ${ }^{+} \mathrm{CD} 4^{+}$ $T$ cells in the indicated EAE groups. ${ }^{*} P \leq 0.05$; NS indicates $P>0.05, n=5$ (mean \pm SEM). (G) FACS analysis of frequencies of IL-4- and IL-17-producing $\mathrm{CD} 4{ }^{+} \mathrm{T}$ cells from dLNs and $\mathrm{mLNs}$ of the indicated groups on day 12 after EAE induction. (H) Average frequencies of IL-4+ CD4 ${ }^{+} T$ cells (left) and IL-4+ IL-17+ $\mathrm{CD}^{+} \mathrm{T}$ cells in the indicated groups ${ }^{*} P \leq 0.05, n=5$ (mean \pm SEM). Two-tailed Student's $t$ test was applied to determine significance.

\section{Results}

Altered expression of Bcl11b in $T$ belper cells results in failure to downregulate GATA3 and IL-4 in Th17-polarized cells. Bcl11b is expressed in $\mathrm{T}$ helper cells (21); however, its role in these cells has never been investigated. We induced in vitro polarization of Bcl11b-deficient $\mathrm{CD} 4^{+} \mathrm{T}$ cells and evaluated lineage-specific cytokines and transcription factors. Our results indicated that while Bcl11b-deficient Th1, Th2, and Th17 cells expressed normal levels of lineage-specific cytokines, Th0 cells produced elevated levels of IL-4 (Supplemental Figure 1, A and B, and data not shown; supplemental material avail- able online with this article; doi:10.1172/JCI70103DS1). Additionally, while T-bet, Roryt, and GATA3 levels were similar to WT under Th1, Th17, and Th2 conditions, respectively, GATA3 was increased in Th0, and remained elevated under Th17 conditions (Supplemental Figure 1, C and D, and data not shown). Furthermore, $B c l 11 b$-deficient polarized Th17 cells exhibited a small population expressing both GATA3 and Roryt (Supplemental Figure 1, C and D). Despite elevated GATA3, Bcl11b-deficient polarized Th17 cells failed to express IL-4 (Supplemental Figure 1A), a result perhaps related to the strict inhibitory conditions in which these cells were 
maintained during Th17 polarization. To test this hypothesis, we repeated the in vitro Th17 polarizations in conditions that lacked inhibitory antibodies for IL-4, which resulted in IL-4 production in $B c l 11 b$-deficient cells, while IL-17 production remained unaffected (Supplemental Figure 1, E and F). A small population of Bcl11bdeficient Th17 cells produced both IL-4 and IL-17 (Supplemental Figure $1, \mathrm{E}$ and $\mathrm{F}$ ). These results suggest that $B c l 11 b$ may play a role in restricting GATA3 and IL-4 expression in Th17 cells.

Th17 cells of EAE Bcl11b/F/dLck-iCre mice express GATA3 without affecting Roryt. Considering the observation that Bcl11b-deficient Th17-polarized cells in vitro not only expressed Roryt and IL-17, but also GATA3 and IL-4, we investigated whether this phenomenon occurred in vivo in the Th17-mediated EAE, using $B c l 11 b^{F / F} /$ dLck-iCre mice, in which $B c l 11 b$ is conditionally ablated in mature $\mathrm{T}$ cells only (27). $\mathrm{CD}^{+} \mathrm{T}$ cells of naive $B c l 11 b^{F / F} / \mathrm{dLck}-\mathrm{iCre}$ mice exhibited elevated GATA3 levels (Figure 1, A and B), whereas Roryt expression was low in both WT and $B c l 11 b^{F / F} /$ dLck-iCre naive mice (Figure 1A). GATA3 levels were downregulated in WT T helper cells following EAE induction (Figure 1C), but remained elevated in T helper cells of EAE Bcl11 $b^{F / F} / \mathrm{dLck}$-iCre mice (Figure 1, C and D) despite overall normal expression of Roryt (Figure 1C). Fur-

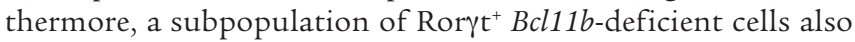
expressed GATA3 (Figure 1, C and D). Not only were GATA3 protein levels elevated, but mRNA levels as well (Supplemental Figure 2A). Considering that $B c l 11 b$ is a transcription factor, we further investigated whether $B c l 11 b$ associates with conserved noncoding sequences within the GATA3 locus in $\mathrm{CD}^{+} \mathrm{T}$ cells, namely with the proximal and the distal GATA3 promoters, using ChiP assays followed by quantitative PCR (qPCR). While proximal promoter is active in T cells, the distal promoter is active in neurons and during Th2 differentiation (29). Our results show that $B c l 11 b$ associates with the proximal GATA3 promoter, but not with the distal promoter, nor with a conserved noncoding sequence in intron 1 , used as a negative control (Supplemental Figure 2B). These results together suggest that $B c l 11 b$ binds to proximal GATA3 promoter to repress its activity during Th17 responses.

Th17 cells of EAE Bcl11b $b^{F / F} / d L c k-i C r e$ mice produce IL-4 without restricting the production of Th17 cytokines. We investigated cytokine production by multiplex cytokine analysis and found that $\mathrm{CD}^{+}$ $\mathrm{T}$ cells from EAE $B c l 11 b^{F / F} / \mathrm{dLck}$-iCre mice produced elevated levels of IL-4 and IL-5 in both the dLNs and mLNs (Supplemental Figure 3). Additionally, IL-17 production was elevated in the mLNs and unchanged in the dLNs of EAE Bcl11 $b^{F / F} / \mathrm{dLck}$-iCre mice (Supplemental Figure 3). FACS analysis further revealed that only the frequency of the $\mathrm{CD} 4^{+} \mathrm{T}$ cells that produced both IL-17 and GM-CSF was significantly reduced in the dLNs of EAE $B c l 11 b^{F / F} / \mathrm{dLck}-\mathrm{iCre}$ mice, but not the frequency of the $\mathrm{CD}^{+} \mathrm{T}$ cells producing IL-17 or GM-CSF alone (Figure 1, E and F). However, the frequency of the $\mathrm{IL}-17^{+} \mathrm{GM}-\mathrm{CSF}^{+} \mathrm{CD} 4^{+} \mathrm{T}$ cell population was increased in the $\mathrm{mLN}$ and was not significantly different from the frequency of IL- $17^{+} \mathrm{GM}-\mathrm{CSF}^{+} \mathrm{CD}^{+} \mathrm{T}$ cells in WT dLNs (Figure 1, E and F), suggesting that the Th17 cells of EAE $B c l 11 b^{F / F} / \mathrm{dLck}$-iCre mice are not defective in the production of Th17 cytokines, but rather the distribution of these cells is shifted from the dLNs to the mLNs. Furthermore, $\mathrm{CD}^{+} \mathrm{T}$ cells from both the dLNs and mLNs of EAE $B c l 11 b^{F / F} /$ dLck-iCre mice produced elevated levels of IL-4 (Figure 1, $\mathrm{G}$ and $\mathrm{H}$ ). In addition, a population that produced both IL-17 and IL-4 was present (Figure 1, G and $\mathrm{H}$ ). $\mathrm{CD}^{+} \mathrm{T}$ cells from nonimmunized $B c l 11 b^{F / F} / \mathrm{dLck}$-iCre mice produced slightly elevated levels of IL-4 but no Th17-associated cytokines (Supplemental Figure 4A).
Together, these findings demonstrate that GATA3 downregulation fails to occur in Thelper cells of EAE $B c l 11 b^{F / F} / \mathrm{dLck}$-iCre mice and consequently these cells express IL-4, despite normal levels of Roryt, IL-17, and GM-CSF.

$B c l 11 b^{F / F} / d L c k$-iCre mice have reduced $E A E$ severity with diminished Th17 cell CNS infiltration in the absence of alterations in the CNS trafficking receptors. During the in vivo analysis of the role of $B c l 11 b$ in Th17 cells, we observed that following EAE induction, the disease scores exhibited by $B c l 11 b^{F / F} / \mathrm{dLck}$-iCre mice were greatly reduced and the onset was delayed (Figure 2A). Histological analysis revealed that at disease peak, EAE $B c l 11 b^{F / F} / \mathrm{dLck}$-iCre mice had considerably less CNS tissue damage and mononuclear infiltrations compared with EAE WT mice (Figure 2B). FACS analysis further revealed that the frequencies and absolute number of $\mathrm{CD} 4^{+} \mathrm{T}$ cells infiltrating the CNS were reduced in EAE $B c l 11 b^{F / F} / \mathrm{dLck}$-iCre mice compared with EAE WT mice (Figure 2, C and G, and Supplemental Figure $5 \mathrm{~A}$ ), and the infiltrating $\mathrm{CD}^{+} \mathrm{T}$ cells produced diminished levels of IL-17 and GM-CSF (Figure 2, D and G, and Supplemental Figure 5A). In addition, the frequency of antigen-specific

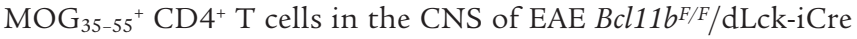
mice was also reduced (Supplemental Figure 5B). This was further demonstrated in EAE Bcl11 b $b^{F / F} /$ dLck-iCre/2D2 mice, which bear the transgenic TCR for $\mathrm{MOG}_{35-55}$ (ref. 30 and Figure 2E), which also produced reduced levels of IL-17 and GM-CSF (Figure 2F). In addition to fewer $\mathrm{CD}^{+} \mathrm{T}$ cells as well as fewer IL-17- and GM-CSFproducing $\mathrm{CD}^{+} \mathrm{T}$ cells, EAE $B c l 11 b^{F / F} / \mathrm{dLck}$-iCre mice had fewer CD $11 b^{+} C D 45^{\text {hi }}$ cells in the CNS (Supplemental Figure 5A), which suggests an overall reduction in neuro-inflammation.

We further evaluated the levels of CCR6 and the integrins $\alpha_{4} \beta_{1}$ (itg $\alpha 4 / \operatorname{itg} \beta 1$ ) and LFA-1 (CD11a and CD18), which are required for migration and infiltration of $\mathrm{T}$ helper cells into the CNS during EAE (31-33), and found that their surface levels were comparable between EAE $B c l 11 b^{F / F} /$ dLck-iCre and WT mice (Figure $2 \mathrm{H}$ ). The percentages of CD $44^{\mathrm{hi}}$ and $\mathrm{CD} 25^{+} \mathrm{CD} 4^{+} \mathrm{T}$ cells were equivalent in the dLNs and elevated in the mLNs on both day 8 and day 12 following EAE induction (Supplemental Figure 6A). Though there were differences in the MFI of CD44 in CD4 ${ }^{+}$ $\mathrm{T}$ cells of EAE $B c l 11 b^{F / F} / \mathrm{dLck}$-iCre and WT mice, these differences were not statistically significant (Supplemental Figure 6B). Levels of CD25 were similar on $\mathrm{CD}^{+} \mathrm{T}$ cells from nonimmunized $B c l 11 b^{F / F} / \mathrm{dLck}$-iCre and WT mice, while CD44 was slightly increased (Supplemental Figure 4B).

These results demonstrate that reduced EAE severity in $B c l 11 b^{F / F} /$ dLck-iCre mice was associated with reduced numbers of Th17 cells infiltrating the CNS, but was not caused by reduced levels of CNS trafficking receptors or diminished activation.

Th17 cells of EAE Bcl11 $b^{F / F} /$ dLck-iCre mice accumulate in the $m L N s$-gut through upregulation of gut-homing receptors without causing overt colitis. The above results indicated that EAE $B c l 11 b^{F / F} / \mathrm{dLck}$-iCre mice have a prevalence of Th17 cells in the mLNs (Figure 1, E and F), which were also found to be enlarged, while the dLNs were reduced in size (Figure $3 \mathrm{~A}$ ). The absolute numbers of $\mathrm{CD}^{+} \mathrm{T}$ cells were reduced in the dLNs and increased in the mLNs of EAE $B c l 11 b^{F / F}$ / dLck-iCre mice (Figure $3 \mathrm{~B}$ ), indicating a redistribution of the cells from the dLNs to the mLNs. Investigation of the small intestine, for which the mLNs are the key priming site, indicated that Peyer patches were also enlarged compared with those in with EAE WT mice (Supplemental Figure 7A), and both Peyer patches and small intestine lamina propria (SILP) had higher frequencies and absolute numbers of $\mathrm{CD}^{+} \mathrm{T}$ cells and activated IL-17-producing $\mathrm{CD} 4^{+}$ 
A

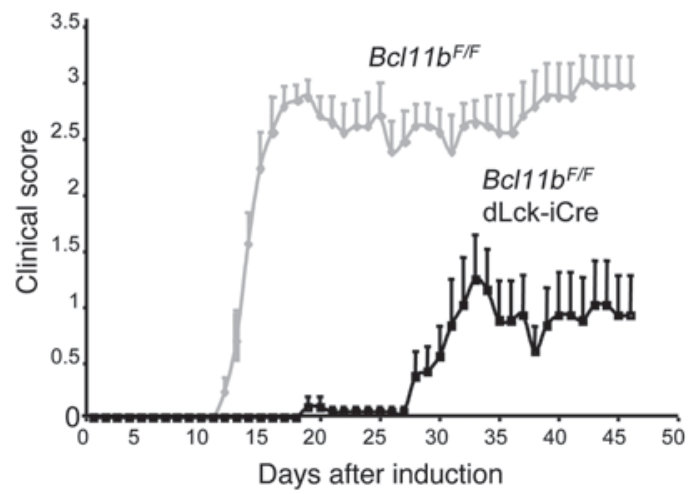

B $B c / 11 b^{F / F}$

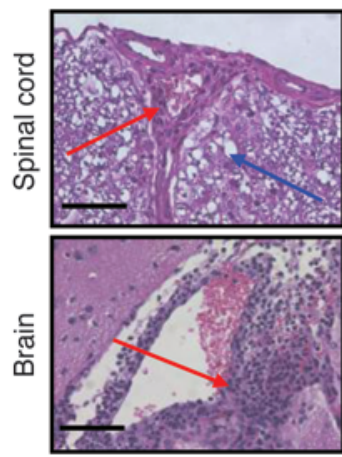

$B c / 11 b^{F / F}$ dLck-iCre

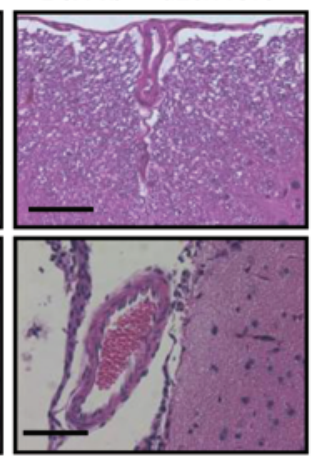

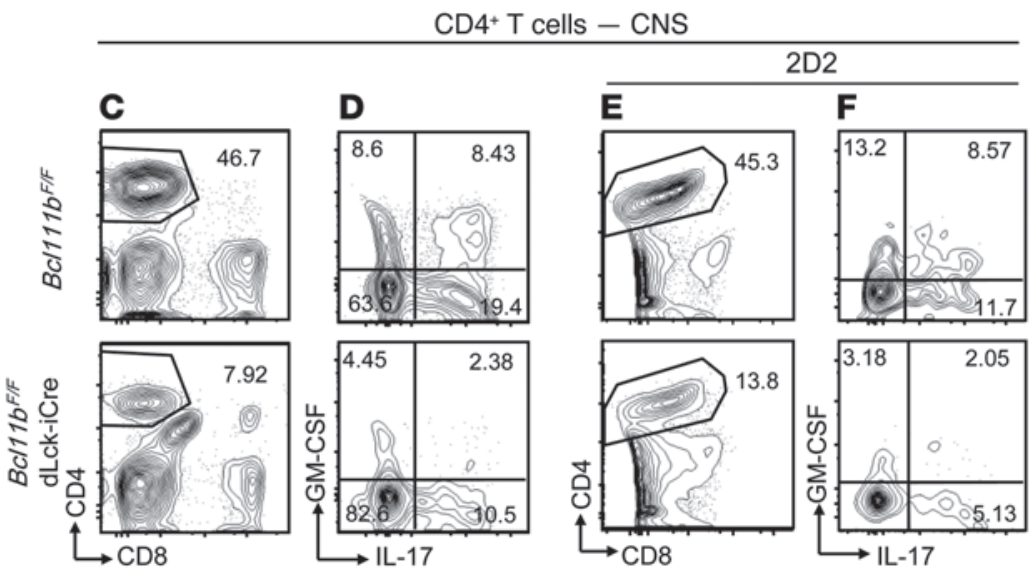

G

H CD4 ${ }^{+} \mathrm{T}$ cells $-\mathrm{dLNs}$
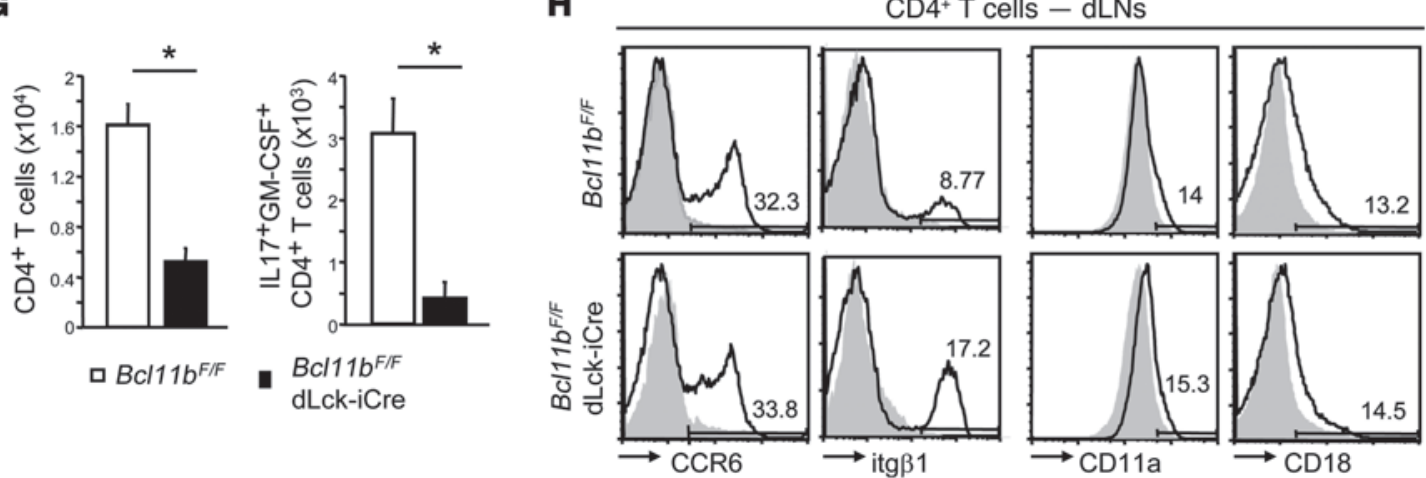

\section{Figure 2}

$B c / 11 b^{F / F / d L c k-i C r e ~ m i c e ~ s h o w ~ d e l a y e d ~ o n s e t ~ o f ~ E A E ~ w i t h ~ r e d u c e d ~ s e v e r i t y ~ c a u s e d ~ b y ~ d i m i n i s h e d ~ C D 4+~} T$ cell infiltration in the CNS, without alteration in CNS trafficking receptors. (A) EAE disease scores of $B c / 11 b^{F / F / d L c k-i C r e ~(b l a c k) ~ a n d ~ W T ~(g r a y) ~ m i c e ~ f o l l o w i n g ~ E A E ~ i n d u c t i o n, ~ a s ~}$ described in Methods. $P \leq 0.05$ starting on day 12, $n=11$ (mean \pm SEM). Data are representative of 4 independent experiments. (B) H\&E staining of spinal cord (top) and brain (bottom) of $B c / 11 b^{F / F / d L c k-i C r e ~ a n d ~ W T ~ m i c e ~} 18$ days after EAE induction. Scale bars: $200 \mu \mathrm{m}$. Red arrows

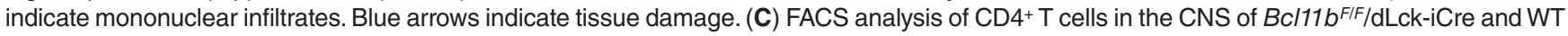
mice on day 18 following EAE induction. (D) Frequencies of IL-17- and GM-CSF-producing CD4+ T cells in CNS on day 18 after immunization.

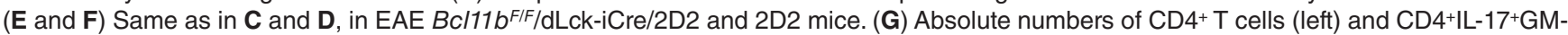
$\mathrm{CSF}^{+}$(right) in the CNS of EAE Bc/11 b ${ }^{F / F} / \mathrm{dLck}$-iCre (black) and WT (white) mice. ${ }^{*} P \leq 0.05, n=6$ (mean \pm SEM). (H) FACS analysis of CCR6, itg $\beta 1$, CD11a, and CD18 levels on CD4 ${ }^{+} T$ cells from dLNs of the indicated EAE mice on day 12 following EAE induction. In all plots, gray shaded area indicates negative control. Two-tailed Student's $t$ test was applied to determine significance.

T cells (Figure 3, C and D, and Supplemental Figure 7, B and C). Even the EAE $B c l 11 b^{F / F} / \mathrm{dLck}-\mathrm{iCre} / 2 \mathrm{D} 2$ mice exhibited an increase in $\mathrm{CD}^{+}$and $\mathrm{IL}-17^{+} \mathrm{CD} 4^{+} \mathrm{T}$ cell accumulation in the Peyer patches and SILP compared with EAE WT 2D2 mice (Supplemental Fig- ure $8 \mathrm{~A})$. Despite the accumulation of $\mathrm{CD}^{+} \mathrm{T}$ cells in the Peyer patches and SILP, EAE $B c l 11 b^{F / F} / \mathrm{dLck}$-iCre mice did not develop overt colitis, as demonstrated by the normal small intestinal tissue histology (Figure $3 \mathrm{E})$. CD4 ${ }^{+} \mathrm{T}$ cells from mLNs, Peyer patches, and 

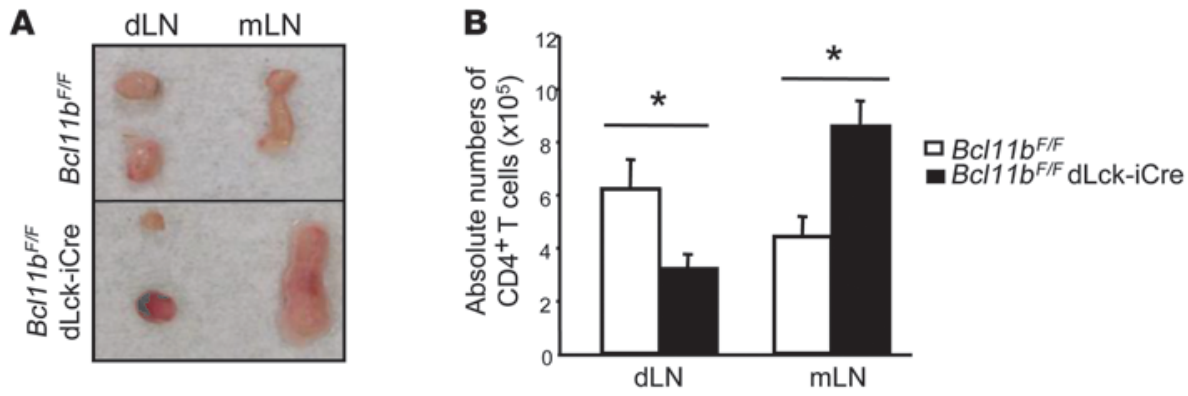

C
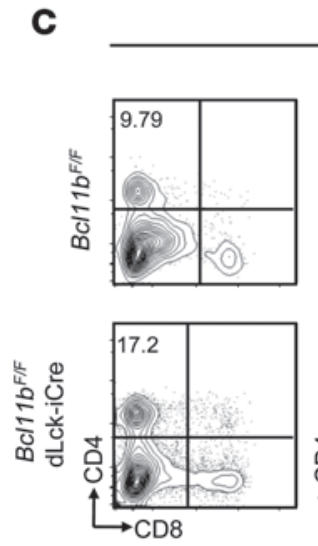

PP

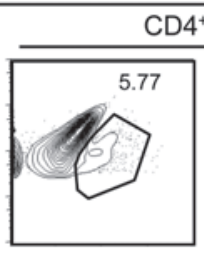

CD4+ $\mathrm{T}$ cells
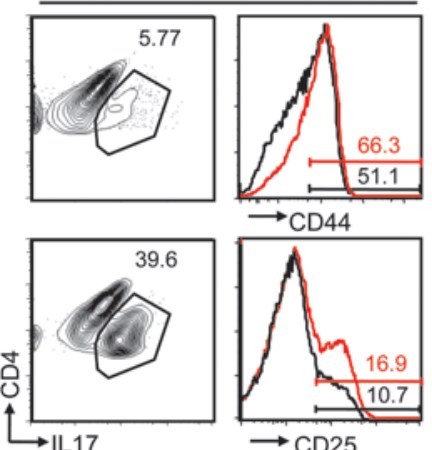
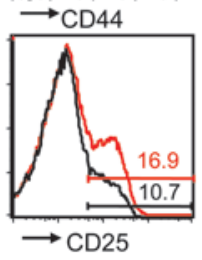

D

SILP
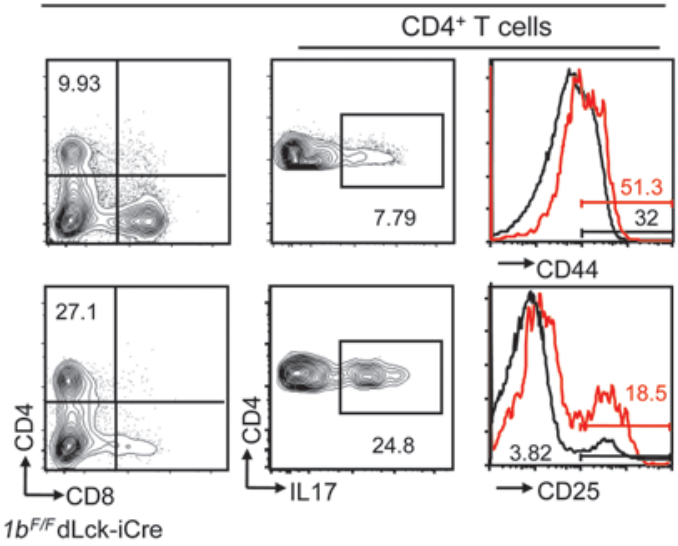

- $B c / 11 b^{F / F}$ - Bcl11 $b^{F / F}$ dLck-iCre

E

$B c / 11 b^{F / F}$

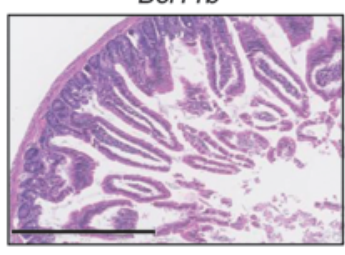

$B c / 11 b^{F / F}$ dLck-iCre

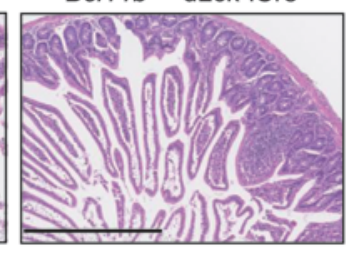

G

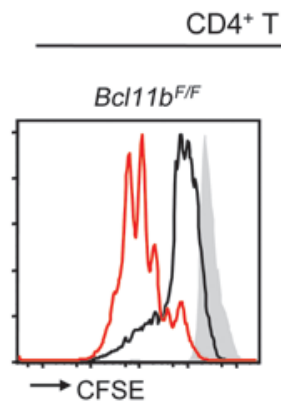

$\mathrm{CD}^{+} \mathrm{T}$ cells
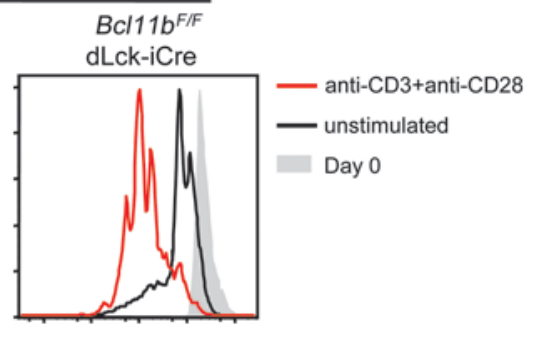

F

$\mathrm{CD}^{+} \mathrm{T}$ cells-EAE
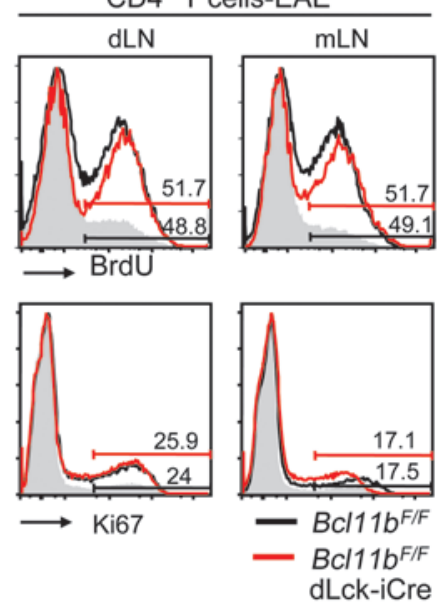

\section{Figure 3}

CD4 ${ }^{+} \mathrm{T}$ cells of EAE Bc/11 $\mathrm{b}^{\mathrm{F} / F / \mathrm{dLck}-i C r e}$ mice accumulate in the enlarged $\mathrm{mLNs}$, Peyer patches, and SILP, proliferate normally, and do not cause overt colitis. (A) Gross anatomy of dLNs and $\mathrm{mLNs}$ from the indicated EAE groups on day 12 after EAE induction. Data are representative of 10 pairs. (B) Absolute numbers of $C D 4^{+} T$ cells in the indicated groups and tissues on day 12 after EAE induction. ${ }^{*} P \leq 0.05 ; n=10$ (mean \pm SEM). (C and D) FACS analysis of CD4, and IL-17, CD44 and CD25 within the CD4+ T cells-gated population in Peyer patches (PP) (C) and SILP (D) on day 18 after EAE induction. (E) H\&E staining of small intestine sections of the indicated groups on day 18 after EAE induction. Scale bars: $400 \mu \mathrm{m}$. Data in C-E are representative of 3 pairs of mice. (F) FACS analysis of BrdU incorporation (top) and Ki67 levels (bottom) in the indicated tissues and groups of EAE mice. Data are representative of 3 pairs of mice. (G) Proliferation of $B c / 11 \mathrm{~b}$-deficient and WT cells evaluated by CFSE dilution. Naive CD4 ${ }^{+} \mathrm{T}$ cells of $B c / 11 \mathrm{~b} / \mathrm{F} / \mathrm{dLck}$-iCre/2D2 and $B c / 11 \mathrm{~b}^{\mathrm{F} / F} / 2 \mathrm{D} 2 \mathrm{mice}$ were labeled with CFSE and incubated for 3 days with plate-bound anti-CD3 and soluble anti-CD28, followed by FACS analysis of CFSE dilution. Red lines indicate CFSE levels following 3 days of treatment; black lines indicate unstimulated cells after 3 days; and gray shaded areas indicate CFSE levels on day 0 . Data are representative of 3 pairs of mice. 
A
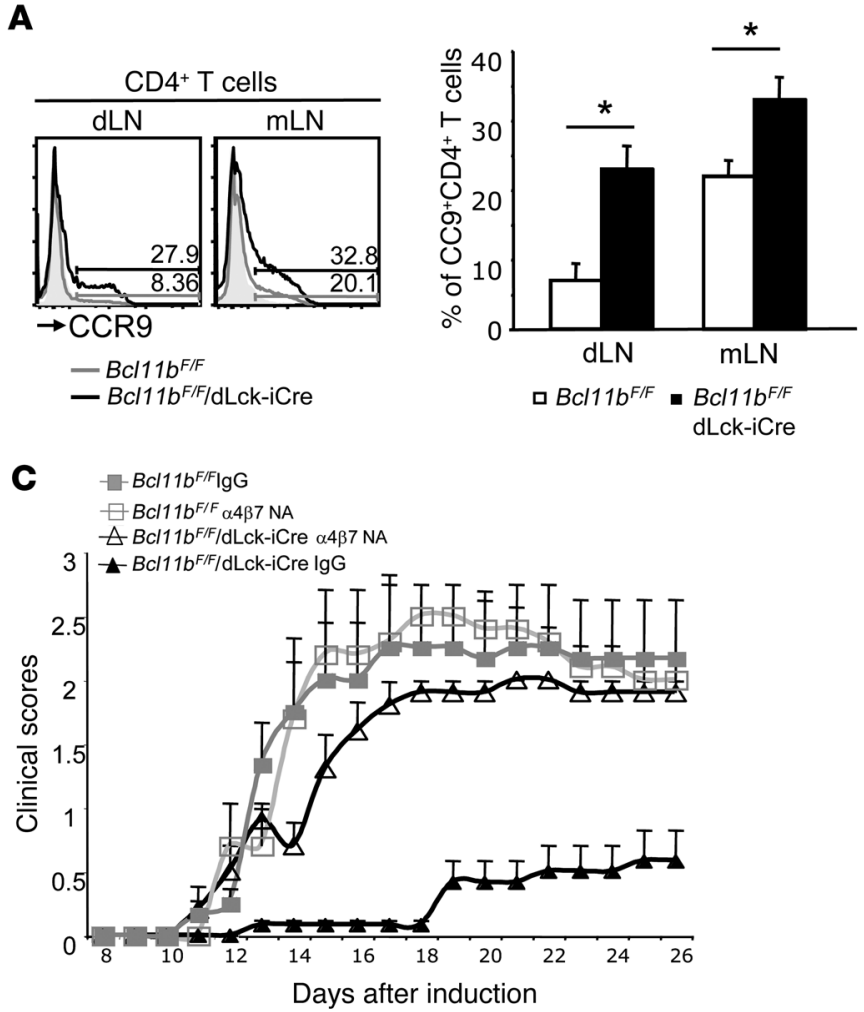

B

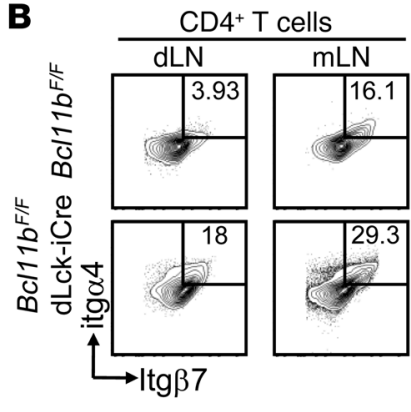

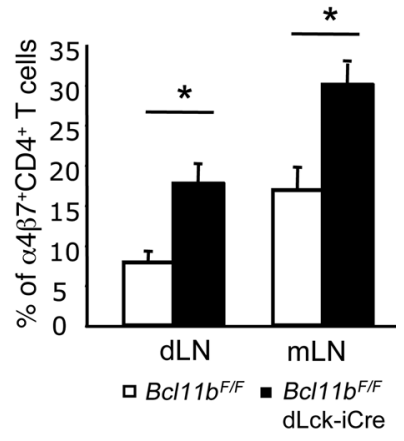

D

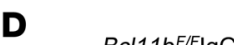

$B c / 11 b^{F / F / d L c k-i C r e ~} \quad B c / 11 b^{F / F / d L c k-i C r e ~}$

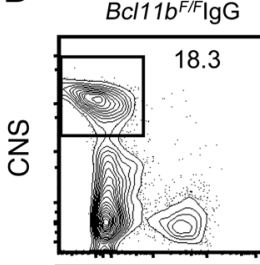

$\operatorname{lgG}$

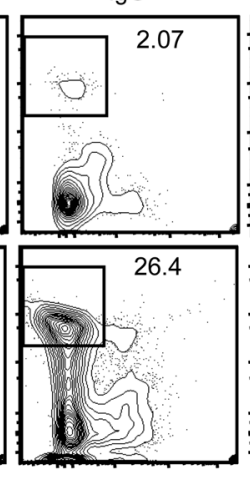

$\alpha 4 \beta 7$ NA

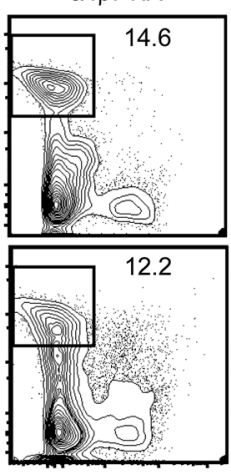

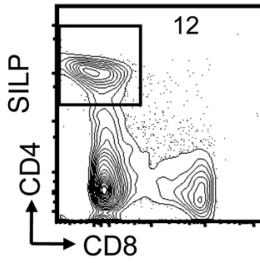

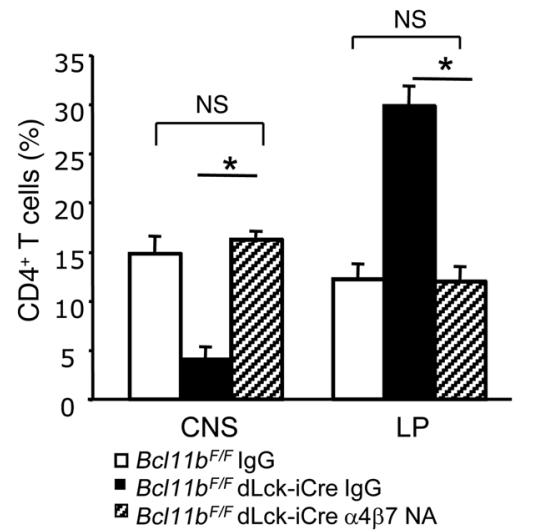

Figure 4

EAE amelioration and preferential gut homing in EAE $B c / 11 b^{F / F / d L c k-i C r e ~ m i c e ~ a r e ~}$ dependent on the upregulation of gut-homing markers on CD4+ $\mathrm{T}$ cells. (A and $\mathbf{B}$ ) FACS analysis of CCR9 (A) and itg4/itg $\beta 7$ (B) on CD4 ${ }^{+}$T cells from dLNs and $\mathrm{mLNs}$ of EAE Bc/11 b/F/dLck-iCre (black) and WT (gray) mice on day 12 after EAE induction. Gray shaded areas indicate negative control. Right panels show average frequencies of CCR9 and $\alpha_{4} \beta_{7}$ levels on CD4 ${ }^{+} \mathrm{T}$ cells from the $\mathrm{dLNs}$ and $\mathrm{mLNs}$ of the indicated EAE mice. ${ }^{*} P \leq 0.05, n=6$ (mean \pm SEM). (C) Clinical scores of the indicated groups of EAE mice treated with anti- $\alpha_{4} \beta_{7}\left(\alpha_{4} \beta_{7} \mathrm{NA}\right)$ or IgG, as described in Methods. $P>0.05$ after day 13 for all, except $B c / 11 b^{F / F / d L c k-i C r e, ~ i n ~ w h i c h ~} P \leq 0.05, n=5$ (mean \pm SEM). Data are representative of 3 independent experiments. (D) FACS analysis of CD4+ $T$ cells in the CNS and SILP of the indicated groups of mice on day 18 following EAE induction. Bottom panel shows average frequency of CD4+ T cells in the CNS and SILP in the indicated groups of mice. ${ }^{\star} P \leq 0.05$; NS indicates $P>0.05, n=4$ (mean \pm SEM).

SILP of EAE Bcl11 $b^{F / F} /$ dLck-iCre mice did not show increased BrdU incorporation and higher Ki67 levels compared with EAE WT mice (Figure 3F and Supplemental Figure 7, D and E), demonstrating that the increase in their number was not caused by increased proliferation. Levels of BrdU incorporation and Ki67 were also equivalent between $B c l 11 b$-deficient CD4 ${ }^{+} \mathrm{T}$ cells and WT in the dLNs (Figure $3 \mathrm{~F}$ ), and overall, the CFSE dilution following in vitro activation of $B c l 11 b$-deficient $\mathrm{CD}^{+}{ }^{+} \mathrm{T}$ cells with anti-CD3/anti-CD28 was equivalent to WT (Figure 3G), suggesting that these cells do not have major alterations in proliferation.

The size of the mLNs and Peyer patches as well as the percentages of CD4 ${ }^{+} \mathrm{T}$ cells in the Peyer patches and SILP were similar between nonimmunized $B c l 11 b^{F / F} /$ dLck-iCre and WT mice (Supplemental Figure 4, C and D), indicating that the observed phenotype is only present following EAE induction. Furthermore, the levels of CD44, $\mathrm{CD} 25$, and Ki67 as well as BrdU incorporation were similar in CD4 ${ }^{+}$ $T$ cells in the dLNs, mLNs, Peyer patches, and SILP in nonimmunized $B c l 11 b^{F / F} /$ dLck-iCre and WT mice (Supplemental Figure 4, E-G).
We further evaluated the integrin $\alpha_{4} \beta_{7}$ and the chemokine receptor CCR9, both known to play roles in gut homing of T cells (34-36), and found them to be upregulated on $\mathrm{CD}^{+} \mathrm{T}$ cells of EAE $B c l 11 b^{F / F} /$ dLck-iCre mice, both in dLNs and mLNs, including in $B c l 11 b^{F / F} / \mathrm{dLck}-\mathrm{iCre} / 2 \mathrm{D} 2$ mice (Figure 4, A and B, and Supplemental Figure 8, B and C), but not on $\mathrm{CD}^{+} \mathrm{T}$ cells of nonimmunized $B c l 11 b^{F / F} / \mathrm{dLck}$-iCre mice (Supplemental Figure 4H).

To demonstrate that the accumulation of the $\mathrm{CD} 4^{+} \mathrm{T}$ cells of EAE $B c l 11 b^{F / F} /$ dLck-iCre mice in the mLNs and gut and the reduced EAE scores were dependent on gut-homing markers, we blocked the function of the integrin $\alpha_{4} \beta_{7}$ using neutralizing antibodies in both EAE $B c l 11 b^{F / F} / \mathrm{dLck}$-iCre and WT mice. We found that $B c l 11 b^{F / F} / \mathrm{dLck}$-iCre mice treated with $\alpha_{4} \beta_{7}$ neutralizing antibodies $\left(\alpha_{4} \beta_{7} \mathrm{NA}\right)$ exhibited disease scores similar to those of both the $\alpha_{4} \beta_{7}$ NA-treated and IgG-treated WT mice (Figure 4C), indicating that upregulation of gut-homing markers is necessary for EAE amelioration in $B c l 11 b^{F / F} / \mathrm{dLck}$-iCre mice. Furthermore, the $\alpha_{4} \beta_{7}$ NA-treated EAE $B c l 11 b^{F / F} /$ dLck-iCre mice exhibited reduced 
A
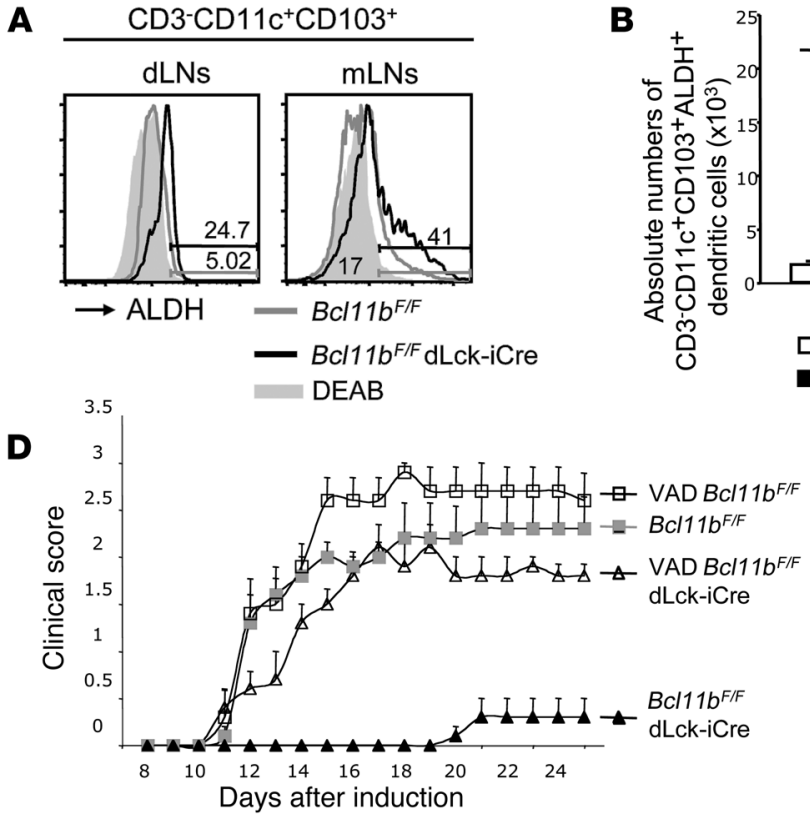

$\mathbf{F}$
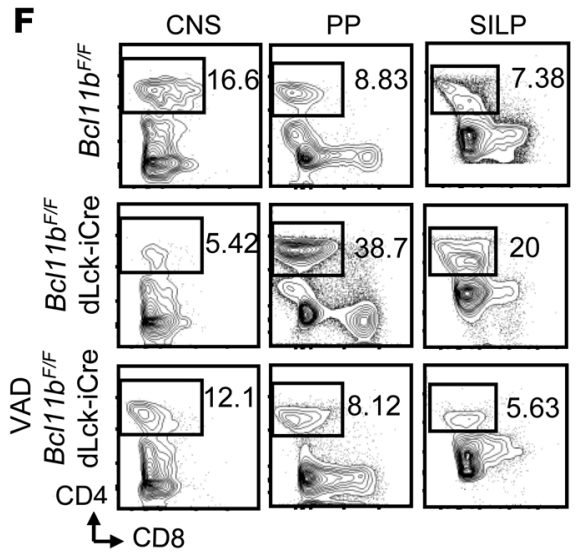

H

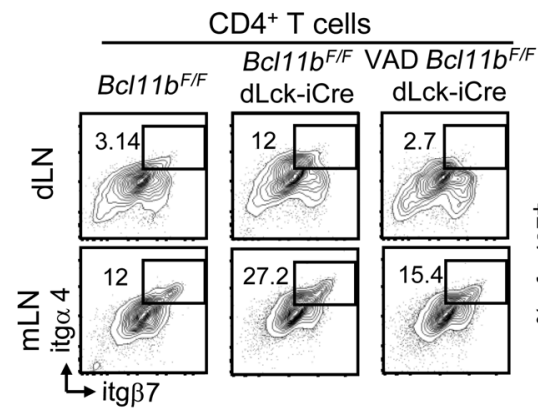

B

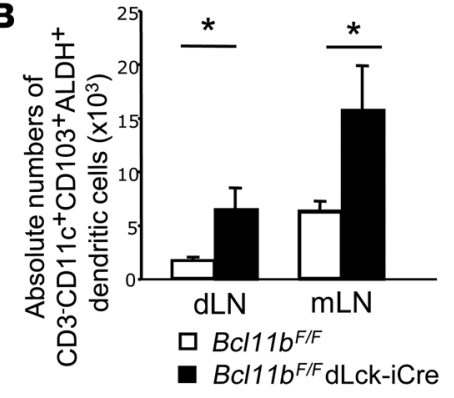

C

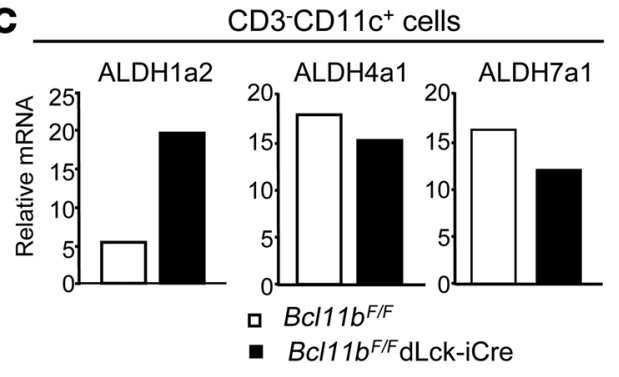

E

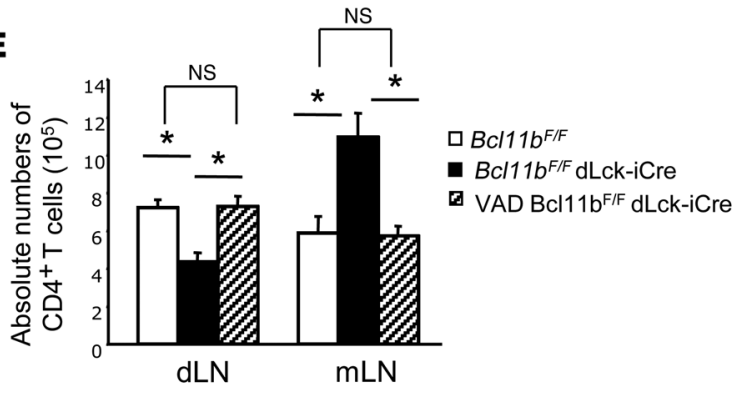

G
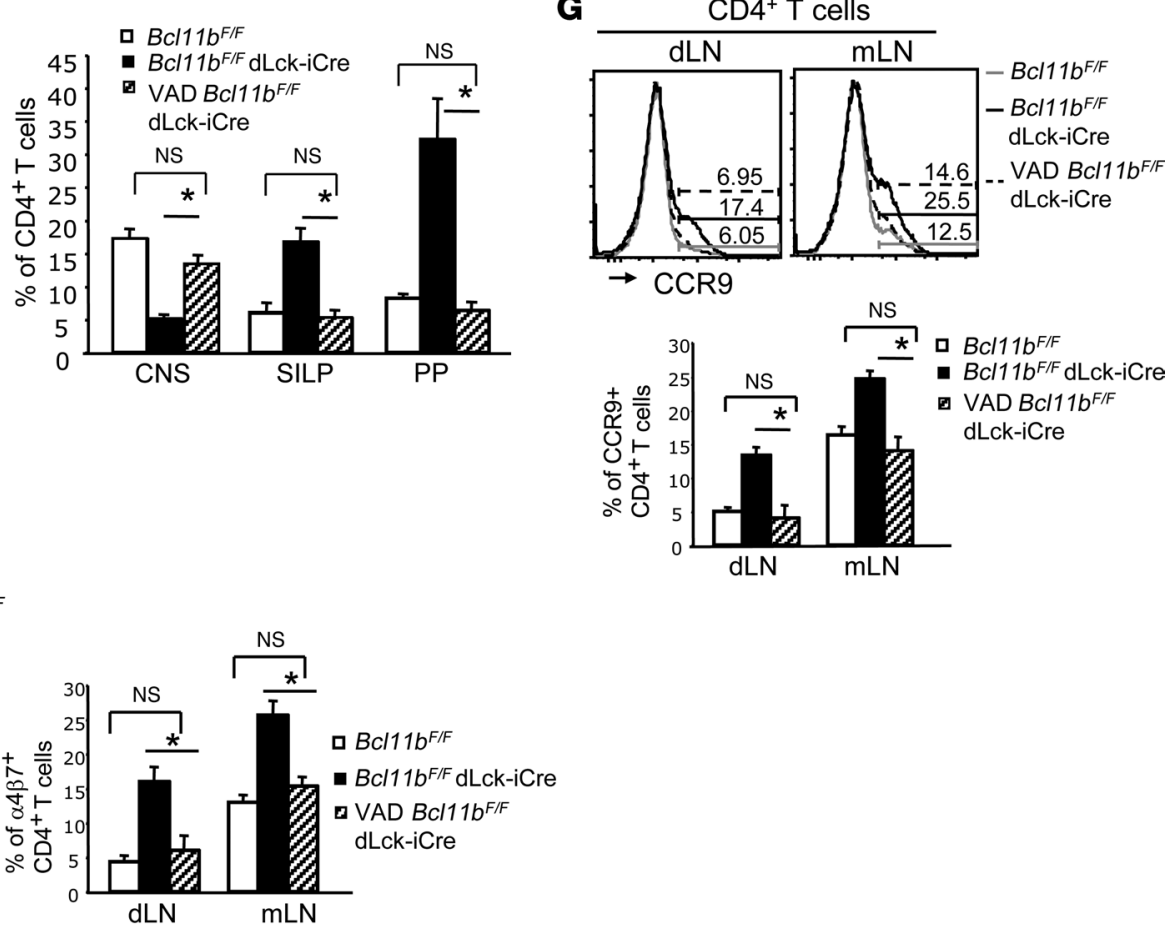

Figure 5

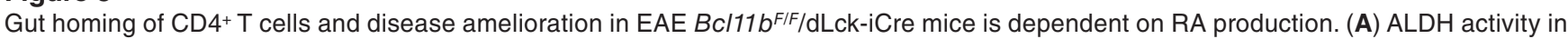
CD3-CD $11 \mathrm{C}^{+} \mathrm{CD} 103^{+}$dendritic cells from the indicated tissues and groups of EAE mice day 12 after EAE induction, evaluated as described in Methods. (B) Absolute numbers of ALDH+CD3-CD11 $\mathrm{C}^{+} \mathrm{CD} 103^{+}$dendritic cells in the indicated tissues and groups of EAE mice. ${ }^{*} P \leq 0.05$; $n=9$ (mean \pm SEM). (C) RT-qPCR for ALDH1a2 (RALDH2), ALDH4a1, and ALDH7a1 in sorted CD3-CD11 ${ }^{+}$dendritic cells of the indicated groups of mice on day 12 after EAE induction. Data are representative of 2 independent experiments with 2 mice per group. (D) EAE scores of the indicated groups of mice on VAD versus normal diet. $P \leq 0.05$ for VAD/BC/11 $b^{F / F / d L c k-i C r e ~ m i c e ~ v e r s u s ~} B c / 11 b^{F / F / d L c k-i C r e ~ m i c e ~ s t a r t-~}$ ing on day $12 ; n=6$ (mean \pm SEM). Data are representative of 3 independent experiments. (E) Absolute numbers of CD4+ T cells from the indicated tissues and groups on day 12 after EAE induction. ${ }^{*} P \leq 0.05$; NS indicates $P>0.05, n=5$ (mean \pm SEM). $(F)$ Frequencies of CD4 ${ }^{+}$ T cells (left) and average frequencies (right) in indicated tissues and groups on day 18 after EAE induction. ${ }^{*} P \leq 0.05$; NS, $P>0.05, n=5$ (mean $\pm \mathrm{SEM}$ ). (G and $\mathbf{H}$ ) FACS analysis (top) and average frequencies (bottom) of CCR9 (G) and $\alpha_{4} \beta_{7}$ integrin $(\mathbf{H})$ on $\mathrm{CD} 4^{+} \mathrm{T}$ cells from the indicated tissues and groups on day 12 after EAE induction. ${ }^{*} P \leq 0.05$; NS, $P>0.05, n=5$ (mean $\pm \mathrm{SEM}$ ). Two-tailed Student's $t$ test was applied to determine significance in all cases. 
accumulation of the $\mathrm{CD}^{+} \mathrm{T}$ cells in the SILP (Figure 4D), while the $\mathrm{CD}^{+} \mathrm{T}$ cell infiltration into the $\mathrm{CNS}$ was similar to that found in IgG-treated controls (Figure 4D). Collectively, these results demonstrate that Th17 cells of EAE $B c l 11 b^{F / F} / \mathrm{dLck}$-iCre mice reside predominantly in the mLNs, Peyer patches, and SILP, rather than being localized in the dLNs and CNS, and this occurs through upregulation of gut-homing receptors. Additionally, these results demonstrate that reduction in EAE severity in $B c l 11 b^{F / F} / \mathrm{dLck}-\mathrm{iCre}$ mice is dependent on upregulation of gut-homing markers.

Gut-homing imprinting on $\mathrm{CD}^{+} \mathrm{T}$ cells of EAE Bcl11b $b^{F / F} / d L c k$-iCre mice is dependent on $R A$ production by dendritic cells. The aforementioned results show that $\mathrm{T}$ helper cells of EAE $B c l 11 b^{F / F} / \mathrm{dLck}$ iCre mice produced increased IL- 4 in both the dLNs and mLNs, maintained IL-17 and GM-CSF production, and upregulated gut-homing markers, which further caused their accumulation in the mLNs-gut rather than in dLNs-CNS. It has been established that dendritic cells differentiated in the presence of GMCSF and IL-4 produce elevated levels of RA (37) due to enhanced expression of the enzyme RALDH2 (or ALDH1a2, where ALDH indicates aldehyde dehydrogenase) $(38,39)$. RA produced by mLNs and gut-associated $\mathrm{CD} 103^{+}$dendritic cells is known to imprint gut-homing specificity on T cells (35). Dendritic cells outside the gut-associated lymphoid tissue (GALT) can also produce RA (40). We evaluated RALDH (ALDH) activity in dendritic cells of EAE Bcl11 $b^{F / F} /$ dLck-iCre mice by flow cytometry, using an aldehyde dehydrogenase-dependent fluorophore (ALDEFLUOR) and found that $\mathrm{CD} 103^{+}$dendritic cells of EAE $B c l 11 b^{F / F} /$ dLck-iCre mice had higher RALDH activity not only in the mLNs, but also in the dLNs (Figure 5A), and there was an increase in the absolute numbers of $\mathrm{CD} 103^{+}$dendritic cells producing ALDH in both the mLNs and dLNs of EAE Bcl11 $b^{F / F} /$ dLck-iCre mice compared with EAE WT mice (Figure 5B). Similar results were seen in EAE $B c l 11 b^{F / F} / \mathrm{dLck}$-iCre mice/2D2 mice (Supplemental Figure 8D). Furthermore, RT-qPCR showed that sorted CD3-CD $11 \mathrm{c}^{+}$dendritic cells of EAE Bcl11 $b^{F / F} / \mathrm{dLck}$-iCre mice expressed elevated levels of ALDH1a2/RALDH2 mRNA, while the levels of mRNAs encoding other aldehyde dehydrogenases, unimportant for RA production, remained the same as in WT cells (Figure 5C). Dendritic cells from nonimmunized $B c l 11 b^{F / F} / \mathrm{dLck}$-iCre mice did not exhibit elevated levels of RALDH activity, demonstrating that this is a consequence of immunization (Supplemental Figure 4I).

To demonstrate that increased RA production is key in the redirection of the Th17 cells from the dLNs and the resultant EAE amelioration in $B c l 11 b^{F / F} /$ dLck-iCre mice, we generated vitamin A-deficient (VAD) Bcl11 $b^{F / F} / \mathrm{dLck}$-iCre mice by provision of a VAD diet, beginning with embryonic day 16 and continuing for the remainder of the study. Previous reports have demonstrated this to be an effective means of RA depletion without causing inanition or death $(38,41)$. EAE VAD/Bcl11 $b^{F / F} /$ dLck-iCre mice presented significantly increased disease scores compared with EAE $B c l 11 b^{F / F} /$ dLck-iCre mice on a regular diet; however, the scores were not significantly different from EAE WT mice or EAE VAD/WT mice (Figure 5D). Furthermore, these mice exhibited a prevalence of $\mathrm{CD}^{+} \mathrm{T}$ cells in the dLNs, and not in the mLNs, similar to EAE WT mice (Figure 5E). In addition, the frequencies of $\mathrm{CD}^{+} \mathrm{T}$ cells in the CNS, Peyer patches, and SILP were similar in EAE VAD $/ B c l 11 b^{F / F} / \mathrm{dLck}$-iCre and EAE WT mice (Figure 5F). Furthermore, $\mathrm{CD}^{+} \mathrm{T}$ cells of EAE $\mathrm{VAD} / B c l 11 b^{F / F} / \mathrm{dLck}$-iCre mice showed reduced CCR9 and $\alpha_{4} \beta_{7}$ levels when compared with EAE $B c l 11 b^{F / F} /$ dLck-iCre mice on a regular diet (Figure 5, G and $\mathrm{H}$ ). These results demonstrate that the diverted migration and disease amelioration in EAE $B c l 11 b^{F / F} / \mathrm{dLck}$-iCre mice are dependent on RA production.

Enhanced $R A$ production in dendritic cells and consequent redirection of T helper cells to the $m L N s$-gut in EAE Bcl11 $b^{F / F} / d L c k$-iCre mice are ultimately dependent on $I L-4$. Considering the abnormal production of IL-4 in EAE Bcl11 $b^{F / F} / \mathrm{dLck}$-iCre mice and the connection between IL-4 and GM-CSF signaling with RA production and the gut-homing phenotype, we further investigated the impact of IL-4 production on (a) RALDH activity in dendritic cells, (b) gut homing of Th17 cells and their relocation, and (c) disease amelioration in $B c l 11 b^{F / F} / \mathrm{dLck}$-iCre mice, using neutralizing antibodies for IL-4 (IL-4NA). We observed that IL-4NA-treated Bcl11 $b^{F / F}$ / dLck-iCre mice had elevated EAE scores compared with IgG-treated $B c l 11 b^{F / F} /$ dLck-iCre mice, but not significantly different from those of IL-4NA- or IgG-treated EAE $B c l 11 b^{F / F}$ mice, respectively) (Figure 6A). Additionally, in EAE IL-4NA-Bcl11 $b^{F / F} / \mathrm{dLck}-\mathrm{iCre} /$ mice, $\mathrm{CD}^{+} \mathrm{T}$ cells accumulated in the dLNs instead of the mLNs (Figure 6B). EAE IL-4NA-Bcl11 $b^{F / F} /$ dLck-iCre mice also presented increased $\mathrm{CD}^{+} \mathrm{T}$ cells infiltrating the $\mathrm{CNS}$ and reduced numbers in SILP and Peyer patches, unlike $B c l 11 b^{F / F} /$ dLck-iCre mice (Figure 6C). RALDH activity was reduced in dendritic cells of EAE IL-4NA$B c l 11 b^{F / F} /$ dLck-iCre mice, concomitant with $\alpha_{4} \beta_{7}$ and CCR9 downregulation on $\mathrm{CD}^{+} \mathrm{T}$ cells, compared with $B c l 11 b^{F / F} / \mathrm{dLck}$-iCre mice (Figure 6, D-F). These results demonstrate that increased RALDH activity in dendritic cells, together with upregulation of gut-homing receptors on $\mathrm{T}$ helper cells and redirected trafficking from the dLNs-CNS route to the mLNs-gut in EAE $B c l 11 b^{F / F} / \mathrm{dLck}$ iCre mice, was dependent on IL-4.

To further demonstrate that Bcl11b-deficient $\mathrm{CD}^{+} \mathrm{T}$ cells induce RA production in dendritic cells through IL-4 and GMCSF, we isolated dendritic cells from $45.1 / \operatorname{Rag}^{-1-}$ mice, antigen pulsed them with $\mathrm{MOG}_{35-55}$, and cocultured these cells in vitro with $\mathrm{CD}^{+} \mathrm{T}$ cells from EAE CD45.2/Bcl11 $b^{F / F} / \mathrm{dLck}-\mathrm{CCre} / 2 \mathrm{D} 2$ or CD 45.2/Bcl11 $b^{F / F} / 2 \mathrm{D} 2$ mice. Dendritic cells cocultured with $B c l 11 b^{F / F} / \mathrm{dLck}-\mathrm{iCre} / 2 \mathrm{D} 2 \mathrm{CD} 4^{+} \mathrm{T}$ cells from both $\mathrm{dLNs}$ and $\mathrm{mLNs}$ upregulated RALDH activity, while dendritic cells cocultured with WT cells exhibited little RALDH activity (Figure 6G). Furthermore, neutralization of IL-4, GM-CSF, or both restricted the upregulation in RALDH activity in dendritic cells cocultured with $B c l 11 b^{F / F} / \mathrm{dLck}$ iCre/2D2 CD4 ${ }^{+} \mathrm{T}$ cells (Figure $6 \mathrm{G}$ ). These results demonstrate that the ability of $B c l 11 b^{F / F} /$ dLck-iCre $\mathrm{CD}^{+} \mathrm{T}$ cells to induce RALDH activity following EAE induction is dependent on IL-4 and GM-CSF.

In support of the implication of GM-CSF in the phenotype observed in the EAE $B c l 11 b^{F / F} / \mathrm{dLck}$-iCre mice comes the immunization of $B c l 11 b^{F / F} / \mathrm{dLck}$-iCre/OT2 mice with $\mathrm{OVA}_{323-339}$ in Th2inducing alum adjuvant, plus Pam3CSK4, a TLR1/2 agonist used to increase the Th2 response. Under these conditions, IL-4 is produced, but not Th17-associated cytokines (Supplemental Figure 9E). RALDH activity was not increased, gut-homing markers were not upregulated, mLNs were not enlarged nor $\mathrm{CD}^{+} \mathrm{T}$ cells accumulated in the Peyer patches or SILP (Supplemental Figure 9, A-D), supporting the idea that IL-4 production per se, in the absence of GM-CSF, is not sufficient for the acquisition of the phenotype.

Collectively, these results demonstrate that increased IL-4 production under Th17 conditions in EAE $B c l 11 b^{F / F} /$ dLck-iCre mice causes increased RA production by dendritic cells, which imprints a gut-homing phenotype and diverts trafficking of Th17 cells to the mLNs-gut. 

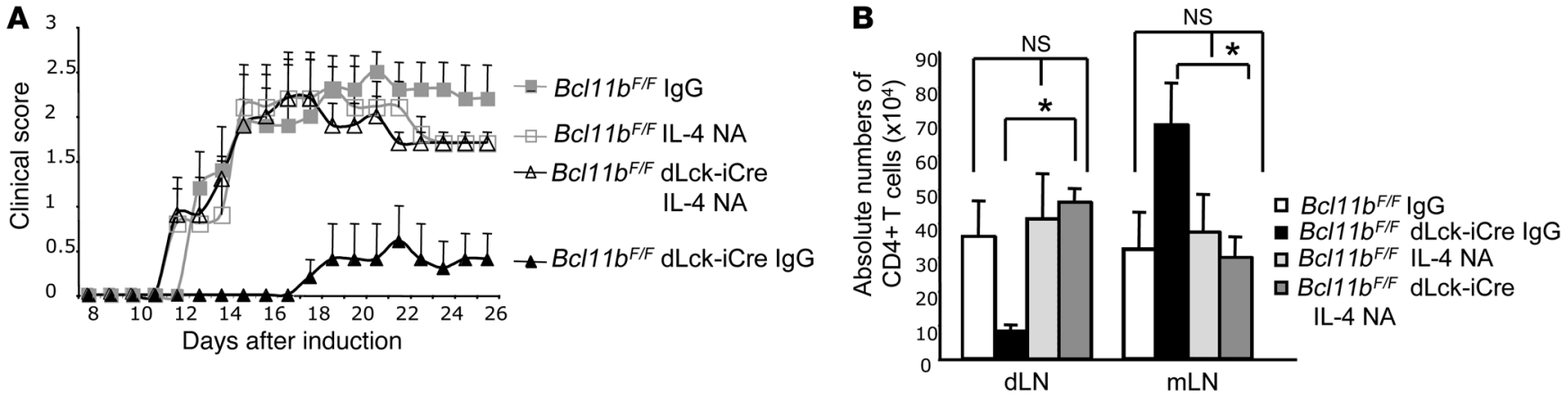

D
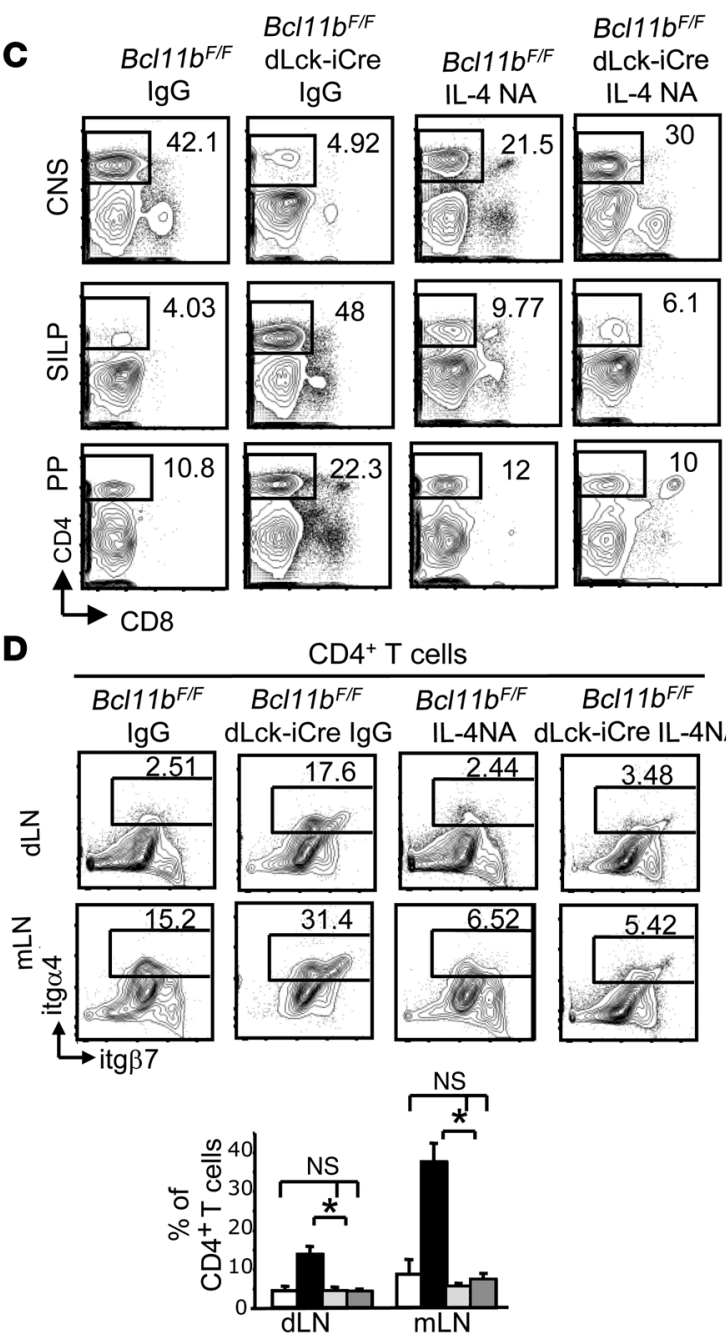

G

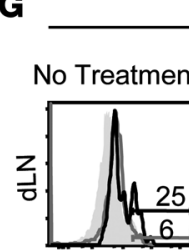
CD3-CD $45.1^{+} \mathrm{CD} 11 \mathrm{c}^{+}$

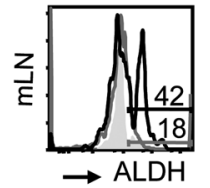

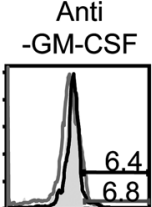

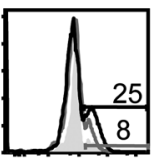

E
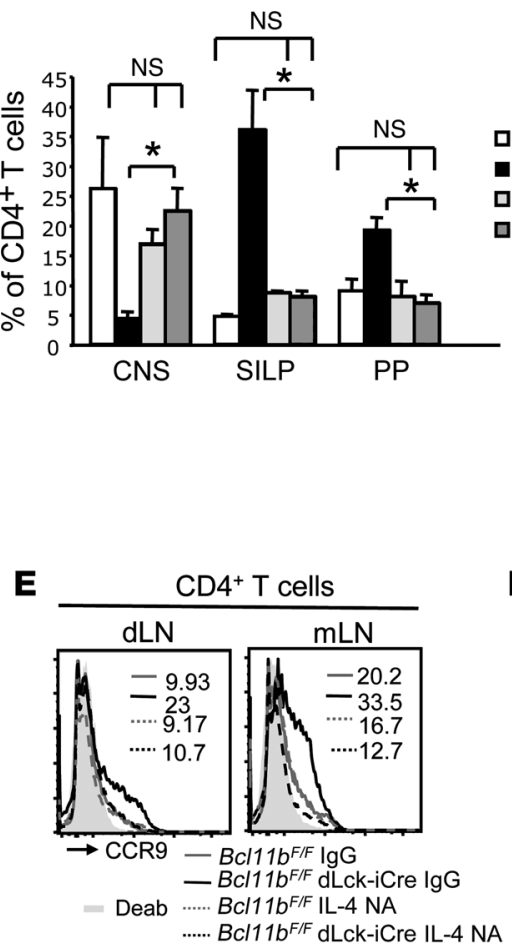

$\mathbf{F}$
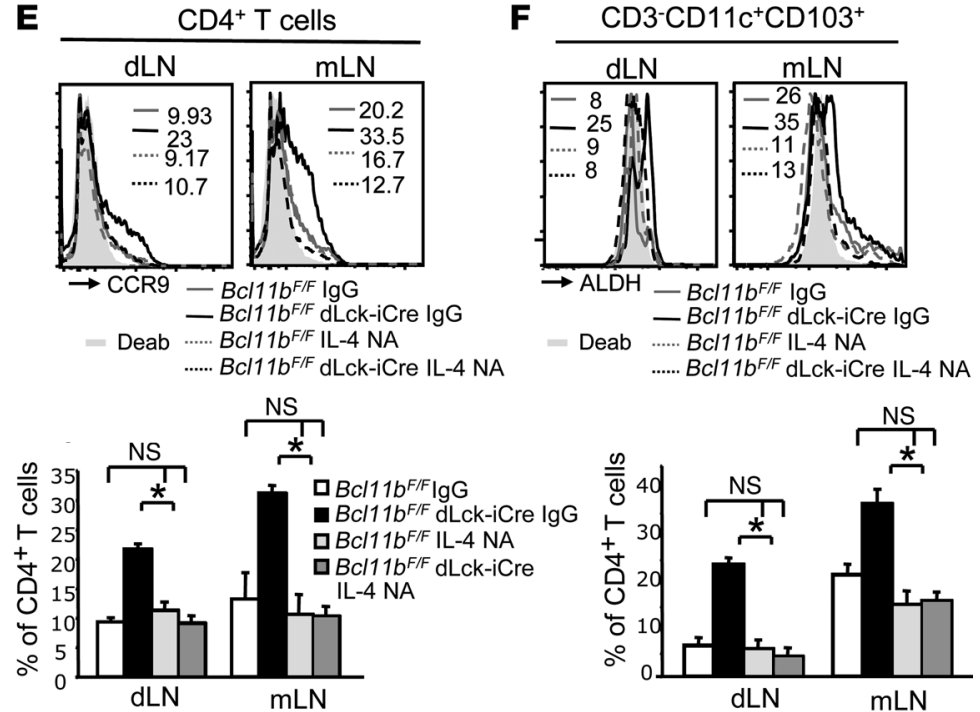


\section{Figure 6}

IL-4 is essential for increased RALDH activity in dendritic cells, upregulation of gut-homing receptors, and $C D 4^{+} T$ cell rerouting and disease amelioration in EAE Bc/11 b/F/dLck-iCre mice. (A) EAE scores of the indicated groups, treated with IL-4NA or IgG. $P \leq 0.05$ for $B c / 11 b^{F / F /}$ dLck-iCre IL-4NA-treated versus IgG-treated, starting with day 12; $n=5$ per group (mean \pm SEM). (B) Absolute numbers of $C D 4^{+} T$ cells in the indicated groups on day 12 after EAE induction. ${ }^{*} P \leq 0.05$; NS, $P>0.05$, $n=6$ (mean \pm SEM). (C) Frequencies by FACS analysis (left) and average frequencies (right) of CD4 ${ }^{+} T$ cells in the indicated tissues and groups on day 18 after induction. ${ }^{*} P \leq 0.05$; NS, $P>0.05, n=4$ (mean \pm SEM). (D and E) $\alpha_{4} \beta_{7}$ integrin (D) and CCR9 (E) evaluated by FACS analysis on $\mathrm{CD}^{+} \mathrm{T}$ cells from dLNs and $\mathrm{mLNs}$ of the indicated groups on day 12 after EAE induction. (F) Flow cytometry analysis of ALDH activity in $\mathrm{CD}^{-} \mathrm{CD} 11 \mathrm{c}^{+} \mathrm{CD} 103^{+}$dendritic cells from $\mathrm{mLNs}$ and $\mathrm{dLNs}$ of the indicated groups on day 12 after EAE induction. (D-F) Lower panels show the average frequencies of the data in the panels above. ${ }^{*} P \leq 0.05$; NS, $P>0.05 ; n=4$ (mean \pm SEM). (G) FACS analysis of ALDH activity in $\mathrm{MOG}_{35-55}$ pulsed dendritic cells from 45.1/Rag2 $2^{-1-}$ mice following 3 days in culture with CD45.2+CD4+ $\mathrm{T}$ cells from dLNs and $\mathrm{mLNs}$ of the indicated groups in conditions that block IL-4, GM-CSF, or both, or without treatment. Data are representative of 3 pairs of mice. Two-tailed Student's $t$ test was applied to determine significance in all cases.

Treatment of EAE WT mice by Th2 immunization or IL-4 prevented progression of $E A E$ due to diverted $\mathrm{CD}^{+} \mathrm{T}$ cell migration. As our findings implicate IL-4 production in the diverted migration of Th17 cells and disease amelioration in EAE $B c l 11 b^{F / F} / \mathrm{dLck}$-iCre mice, we investigated whether the relocation of $T$ helper cells could be induced in EAE WT mice via IL-4 treatment or the induction of IL-4 production by immunization in Th 2 conditions. We treated EAE WT mice with IL-4 and demonstrated that this treatment caused a dramatic reduction in the severity of EAE (Figure 7A), concomitant with decreased $\mathrm{CD} 4^{+} \mathrm{T}$ cells in the dLNs and CNS and enhanced accumulation of $\mathrm{CD}^{+}{ }^{+} \mathrm{T}$ cells in the mLNs, SILP, and Peyer patches (Figure 7, B and C). Additionally, we observed that $\mathrm{CD}^{+} \mathrm{T}$ cells from WT mice treated with IL-4 upregulated guthoming receptors on both $\mathrm{dLN}$ and $\mathrm{mLN} \mathrm{CD}^{+} \mathrm{T}$ cells (Figure 7, D and $\mathrm{E}$ ) and the dendritic cells of these mice had elevated RALDH activity when compared with untreated EAE WT mice (Figure 7F).

Furthermore, treatment of EAE WT mice with $\mathrm{MOG}_{35-55}$ in alum and Pam3CSK4 (MOG/alum) caused T helper cells to produce elevated IL-4 levels without a dramatic block in IL-17 and GM-CSF production (Supplemental Figure 10, A and B). Similarly, GATA3 was upregulated with no change in Roryt (data not shown). Compared with treatment with alum alone, MOG/alum-treated WT mice presented significantly reduced EAE clinical scores (Figure 8A), reduced infiltration of $\mathrm{CD}^{+} \mathrm{T}$ cells in the CNS, and increased percentages of $\mathrm{CD}^{+} \mathrm{T}$ cells in Peyer patches and SILP, but with no overt inflammation in intestinal tissue (Figure 8, D and I). Additionally, the dLNs were smaller, while $\mathrm{CD}^{+} \mathrm{T}$ cells accumulated in the mLNs, which were enlarged along with the Peyer patches (Figure 8, B and C). CCR6 expression remained unaffected, while $\alpha_{4} \beta_{7}$ and CCR9 were upregulated (Figure 8, E-G), similar to EAE $B c l 11 b^{F / F} / \mathrm{dLck}$-iCre mice, while dendritic cells exhibited increased RALDH activity (Figure $8 \mathrm{H}$ ). These findings demonstrate that the induction of IL-4 production during EAE in WT mice did not substantially affect GM-CSF or IL-17 production, but instead caused increased RALDH activity in dendritic cells, thereby imprinting $\mathrm{CD}^{+} \mathrm{T}$ cells with gut-homing specificity. This treatment ultimately resulted in reduced EAE severity, thus providing a potentially new and simple therapeutic avenue for MS.

\section{Discussion}

$\mathrm{T}$ helper cell subsets drive functionally specialized immune responses following infection. It was believed that distinct $\mathrm{T}$ helper subsets exist independently and the presence of alternative lineage cytokines could block differentiated T helper cells (reviewed in ref. 3 ). Recent evidence suggests that $T$ helper subsets have increase plasticity in vivo (reviewed in refs. $3,10,11$ ). We demonstrate that during EAE, Bcl11b-deficient Th17 cells failed to downregulate GATA3 and consequently produced IL-4. Unexpectedly, and in contrast to published with vitro results (9), expression of GATA3 and IL-4 did not block Roryt, IL-17, and GM-CSF production, and resulted in a mixed Th17/Th2 phenotype in cells deficient in $B c l 11 b . B c l 11 b$ is a transcriptional regulator that can act as either a transcriptional repressor or activator, dependent upon which histone-modifying complexes are recruited to the promoter. Our previous studies demonstrate that $B c l 11 b$ can recruit the NuRD complex to induce transcriptional repression by histone deacetylase activity (19) or, conversely, $B c l 11 b$ can recruit the p300 histone acetyltransferase to induce transcriptional activation (20). Furthermore, it is well established that the promoters of $\mathrm{T}$ helper lineagespecific transcription factors have bivalent histone modifications, which maintain chromatin available for activation (42). Therefore, a transcription factor as flexible as $B c l 11 b$ is likely to modulate $\mathrm{T}$ helper lineage determination. Whether $B c l 11 b$ plays additional roles in T helper lineage commitment is currently under investigation.

In our study, we demonstrated that Bcl11b deficiency led to IL-4 production in the dLNs and mLNs, while IL-4 production did not block IL-17 and GM-CSF expression; however, IL-4 in concert with GM-CSF had a major impact on T helper cell migration, specifically rerouting the cells from the dLNs-CNS path to the mLNs-gut, ultimately resulting in reduced EAE severity (model in Supplemental Figure 11A). Aberrant IL-4 production along with GM-CSF caused upregulation of RALDH activity in dendritic cells not only of mLNs, but also of the dLNs, and expression of gut-homing receptors on $\mathrm{CD}^{+}{ }^{+} \mathrm{T}$ cells in the $\mathrm{mLNs}$ as well as the dLNs of EAE $B c l 11 b^{F / F} /$ dLck-iCre mice, causing rerouting of the $\mathrm{CD}^{+} \mathrm{T}$ cells. Importantly, this phenomenon occurred even in conditions in which Bcl11b was not ablated in T helper cells, specifically in EAE WT mice treated with either IL-4 or MOG/alum to induce IL-4 production (model in Supplemental Figure 11B). Accordingly, induction of alternative lineage cytokine production, without blocking per se lineage-specific cytokines, appears to have a major impact on other physiological components, such as immune cell trafficking (models in Supplemental Figure 11, A and B). Importantly, inhibition of RA and IL-4 production or gut-homing receptors in $B c l 11 b^{F / F} / \mathrm{dLck}$-iCre mice resulted in a blockade of gut-homing migration and restoration of EAE symptoms, suggesting that protection from EAE arises solely due to altered T helper trafficking and Bcl11b-deficient Th17 cells have pathogenic potential. We did not observe dramatic increases in IL-4 production in mice treated with alum alone, most likely due to the lack of Pam3CSK4 treatment and perhaps due to the fact that the antigen depot from $\mathrm{MOG}_{35-55} / \mathrm{CFA}$ immunization had diminished below levels that could stimulate immune responses and/or remained associated with CFA.

GM-CSF was recently demonstrated to be a critical cytokine for EAE pathology $(5,6)$; however, GM-CSF has multiple roles in the immune system, such as stimulating, together with IL-4, dendritic cell maturation and induction of RA production (37). We propose that in EAE $B c l 11 b^{F / F} / \mathrm{dLck}$-iCre mice, as well as in EAE WT mice 
A

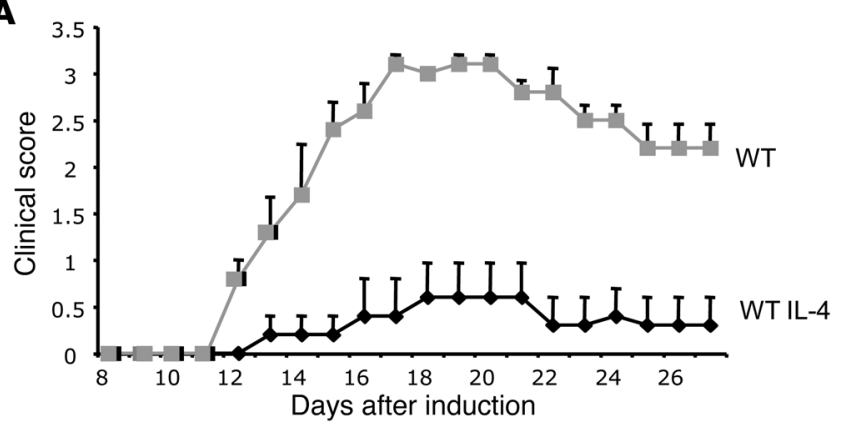

B

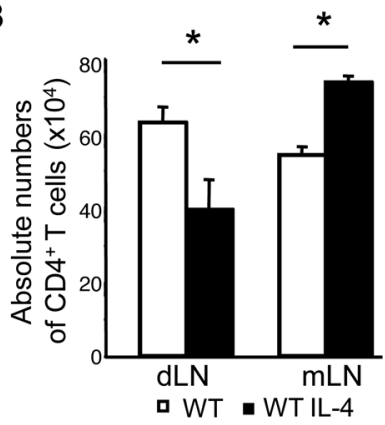

C
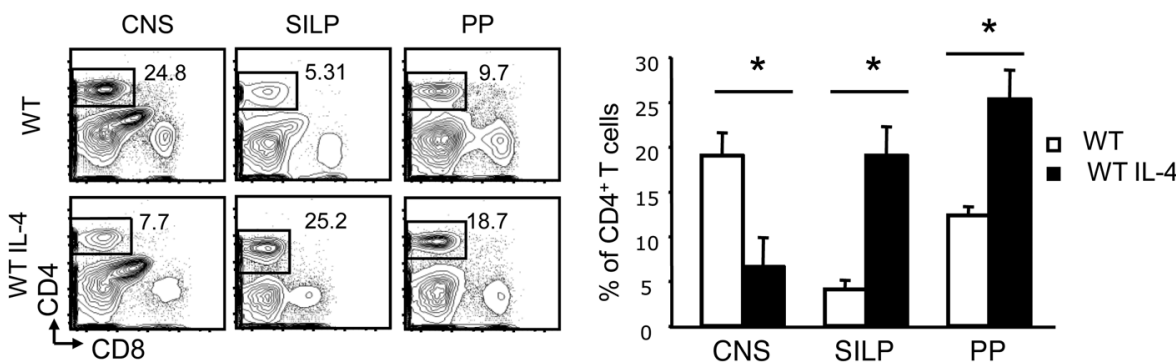

D

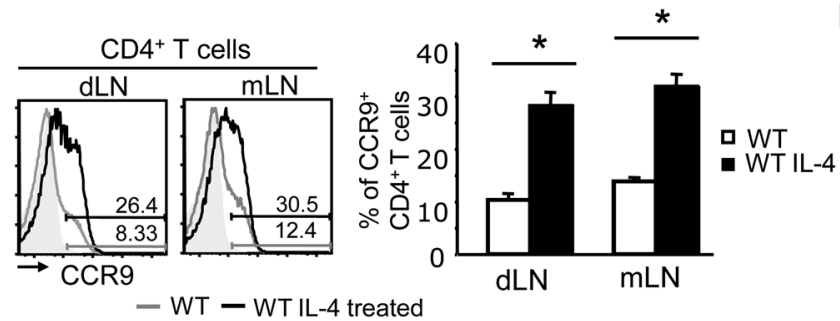

E
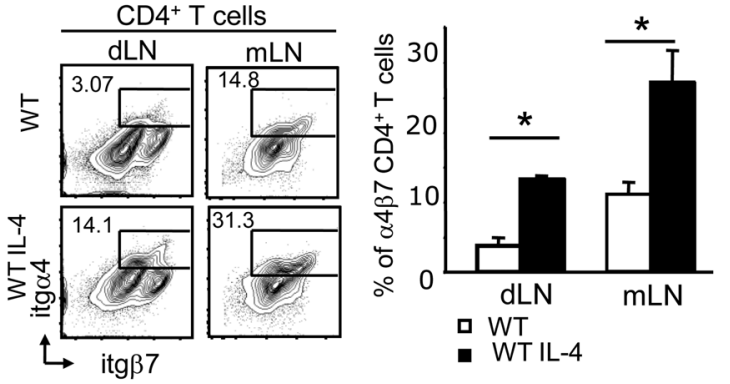

F

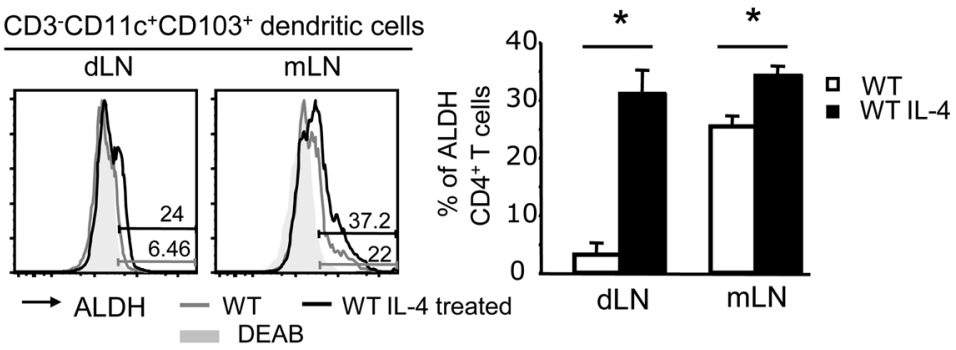

\section{Figure 7}

Treatment of EAE WT mice with IL-4 results in disease amelioration, CD4+ T cell migration to the gut, increased RALDH activity in dendritic cells, and upregulation of gut-homing markers on CD4+ $T$ cells. (A) EAE scores in WT mice treated with recombinant IL-4 (WT IL-4) versus salinetreated controls (WT). $P \leq 0.05$ after day 12; $n=5$ (mean \pm SEM). (B) Absolute numbers of CD4+ $4^{+}$cells from dLNs and mLNs of the indicated groups on day 12 after EAE induction. ${ }^{*} P \leq 0.05 ; n=4$ (mean \pm SEM). (C) FACS analysis of frequencies (left) and average frequencies (right) of CD4 ${ }^{+} \mathrm{T}$ cells in the CNS, SILP, and Peyer patches in the indicated groups on day 18 following EAE induction. ${ }^{*} P \leq 0.05, n=4$ (mean \pm SEM). (D) FACS analysis of CCR9 levels on CD4 ${ }^{+} T$ cells (left) and average frequencies of CCR9 ${ }^{+} C D 4^{+} T$ cells (right) from dLNs and mLNs of the indicated groups. ${ }^{*} P \leq 0.05, n=4$ (mean \pm SEM). (E) FACS analysis of $\alpha_{4} \beta_{7}$ levels on CD4 ${ }^{+} \mathrm{T}$ cells (left) and average frequencies of $\alpha_{4} \beta_{7}{ }^{+} C D 4^{+} \mathrm{T}$ cells (right) from dLNs and $\mathrm{mLNs}$ of indicated groups. ${ }^{*} P \leq 0.05, n=4$ (mean $\pm \mathrm{SEM}$ ). (F) ALDH activity in dendritic cells from dLNs and $\mathrm{mLNs}$ of EAE mice treated with IL-4 or saline. Right panel shows average frequencies of ALDH-producing cells. ${ }^{*} P \leq 0.05, n=4$ (mean \pm SEM). The gray shaded areas represent DEAB-treated samples. Two-tailed Student's $t$ test was applied to determine significance in all cases.

treated with IL-4 or MOG/alum, IL-4 acts in conjunction with GMCSF and induces increased RALDH2 expression and RA production by dendritic cells, followed by gut imprinting on $\mathrm{T}$ helper cells and their diverted migration from the dLNs-CNS route to the mLNs-gut (models in Supplemental Figure 11, A and B), rather than simply exerting a suppressive effect, as previously thought (43). RA itself was shown to be beneficial in EAE treatment (44), believed to be suppressive and able to shift the balance between Th17/iTreg cells (45), due in part to the high plasticity of these subsets (10). However, recent data suggest that iTreg cell generation and Th17 cell inhibition occur at high RA concentrations, while low physiological concentrations of RA support Th17 differentiation $(46,47)$. No 
A

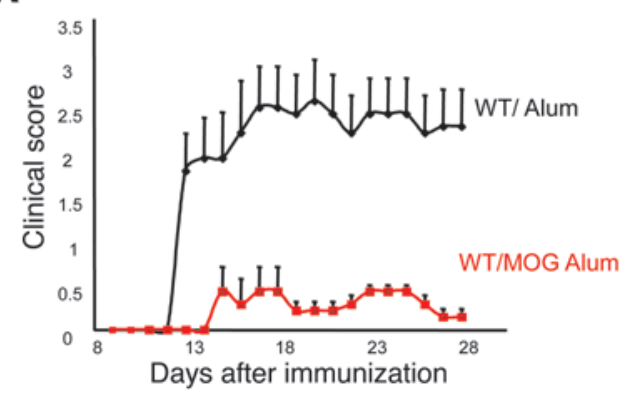

D
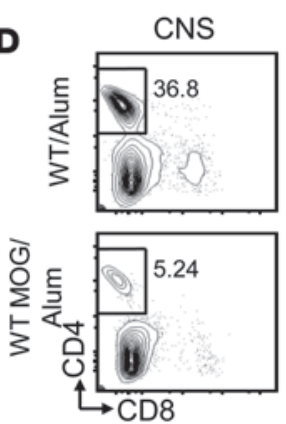

$F$

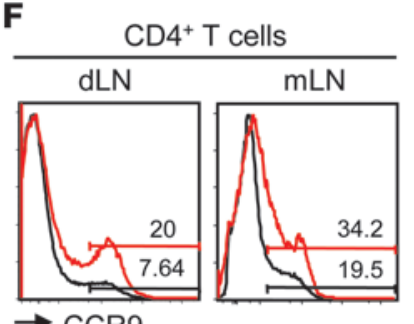

CCR9
B

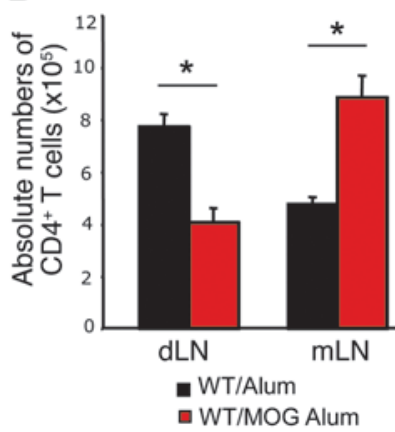

Peyer's
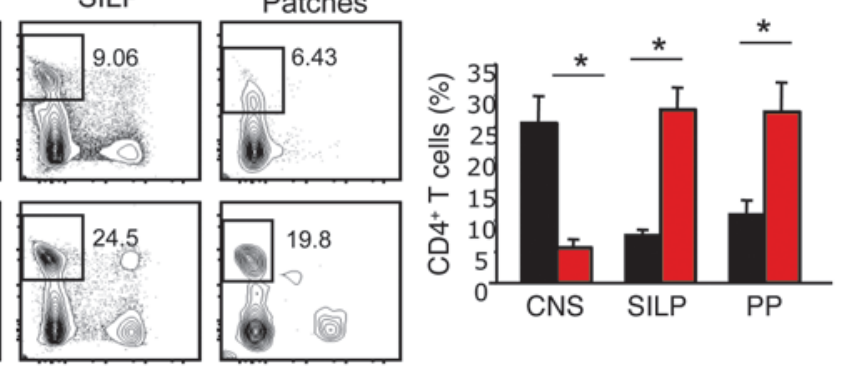
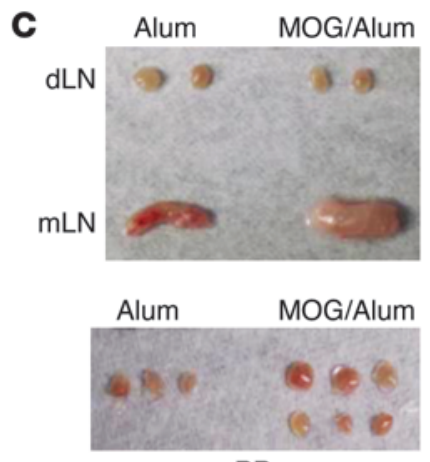

PP

E

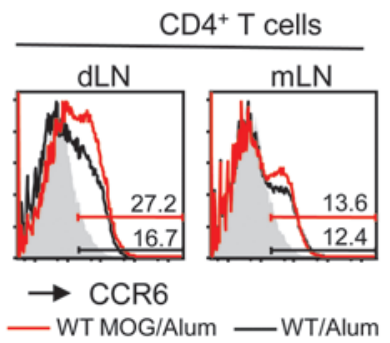

G

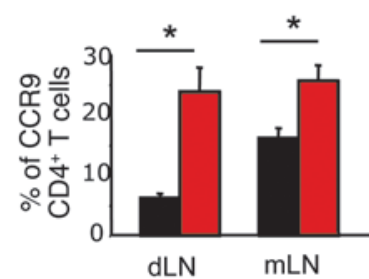

dLN $\quad \mathrm{mLN}$

—WT MOG/Alum —WT/Alum

H

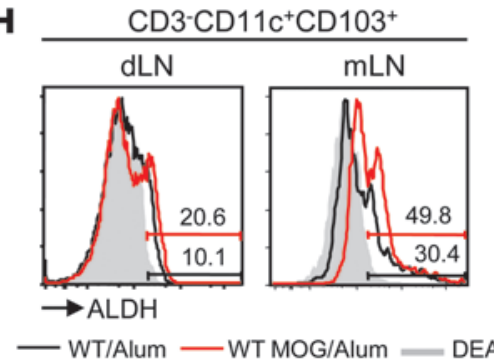

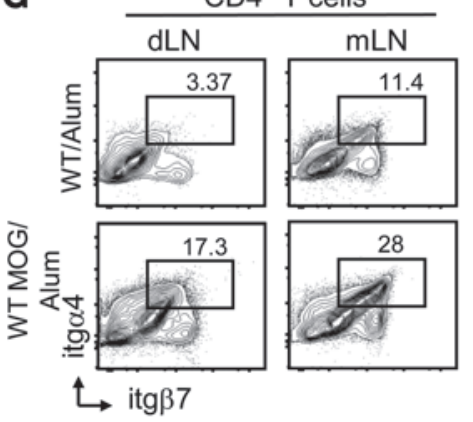

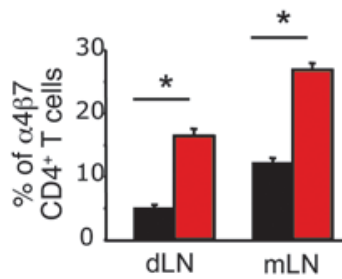

I
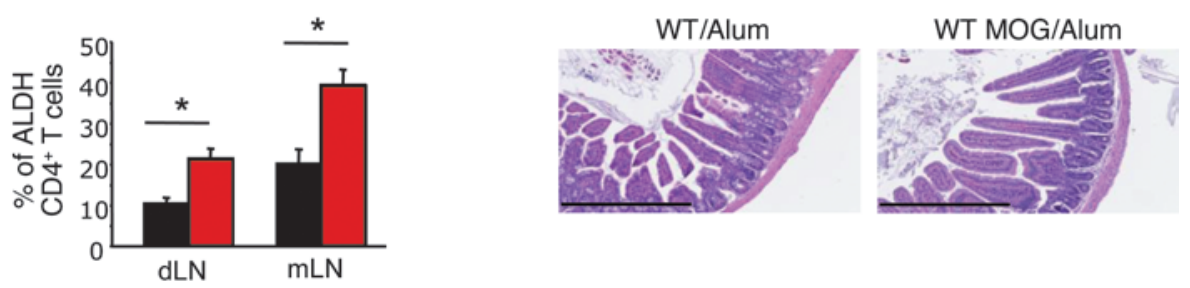

Figure 8

Treatment of EAE WT mice under Th2 conditions reduces clinical scores, causing redistribution of the CD4+ ${ }^{+}$cells from the dLNs-CNS route to the mLNs-gut without overt colitis. (A) EAE scores in WT mice treated with either MOG $_{35-55}$ in alum plus Pam3CSK4 (WT MOG/alum) or alum alone (WT/alum) on day 7 after EAE induction. $P \leq 0.05$ after day 12; $n=7$ (mean \pm SEM). Data are representative for 3 experiments. (B) Absolute numbers of CD4+ $\mathrm{T}$ cells from dLNs and $\mathrm{mLNs}$ from the indicated groups on day 12 after EAE induction. ${ }^{*} P \leq 0.05, n=6$ (mean $\pm \mathrm{SEM}$ ) (C) Gross anatomy of dLNs, mLNs, and Peyer patches on day 12 after EAE induction in the indicated groups. (D) FACS analysis of frequencies (left) and average frequencies (right) of CD4 ${ }^{+} T$ cells in the CNS, Peyer patches, and SILP from the indicated groups on day 18 after EAE induction. ${ }^{*} P \leq 0.05, n=5$ (mean \pm SEM). (E) CCR6 levels on CD4+ $T$ cells from dLNs and mLNs of the indicated groups of EAE mice. (F and $\left.\mathbf{G}\right) \mathrm{CCR} 9$ and $\alpha_{4} \beta_{7}$ levels on CD4 ${ }^{+} \mathrm{T}$ cells from dLNs and mLNs of the indicated groups of EAE mice. ${ }^{*} P \leq 0.05, n=5$ (mean \pm SEM). (H) ALDH activity in dendritic cells from dLNs and $\mathrm{mLNs}$ of the indicated groups. ${ }^{*} P \leq 0.05, n=5$ (mean $\pm \mathrm{SEM}$ ). (I) H\&E staining of small intestine sections of the indicated groups of mice on day 30 after EAE induction. Scale bars: $500 \mu \mathrm{m}$. Data are representative of 3 pairs of mice. Significance was determined by 2 -tailed Student's $t$ test in all cases. 
increase in the numbers of Treg cells was found in EAE Bcl11 $b^{F / F} /$ dLck-iCre mice (data not shown), supporting the notion that therapeutic effect of RA occurs primarily through the diverted migration of the Thelper cells away from the CNS.

Our finding that $\mathrm{T}$ helper cells migrated to the gut despite expression of CCR6 and integrins required for CNS homing suggests that expression of gut-homing receptors can override other migration signals. CCL25, the chemokine for CCR9, is constitutively produced in the gut (48), and thus, once CCR9 is expressed, $\mathrm{T}$ cell homing toward this site may become the default trafficking pathway. CCR9 is important in homing of T cells to SILP, while integrin $\alpha_{4} \beta_{7}$ has a broader impact, homing $\mathrm{T}$ cells to the mLNs, Peyer patches, and SILP $(36,49)$. While expression of CCR6 is critical for CNS migration of T helper cells in EAE, it promotes migration of T helper cells to any sites where IL-17 is produced, related to increased CCL20 (50). Thus, it is possible that this pathway may also contribute to the migration of Th17 cells to the gut in EAE $B c l 11 b^{F / F} / \mathrm{dLck}$-iCre mice and MOG/alum-treated EAE WT mice, as these cells still express CCR6.

Inappropriate $\mathrm{T}$ cell migration would have disastrous effects in the clearance of pathogens during an infection, resulting in inefficient immune responses. However, in autoimmune diseases, redirection of cells to other sites can result in protection, as seen in EAE $B c l 11 b^{F / F} / \mathrm{dLck}$-iCre mice or IL-4- or MOG/alum-treated EAE WT mice. Interestingly, despite increased numbers of IL-17-producing cells infiltrating SILP, neither EAE $B c l 11 b^{F / F} /$ dLck-iCre mice nor MOG/alum-treated EAE WT mice developed overt colitis. Our findings regarding the absence of colitis are in line with the findings demonstrating that IL-17+ $\mathrm{T}$ helper cells can be protective in colitis by inhibiting the development of Th1 responses in the gut (51). Previous studies have shown that mechanisms are in place to limit pathogenicity of these cells, such as acquisition of a regulatory phenotype and elimination via the intestinal lumen, providing protection from inflammatory conditions (50). Further supporting this idea, therapy with an antibody against IL-17 in Crohn disease patients caused high rates of adverse reactions or was ineffective (52). Recent data also suggest that the presence of RA protects against the development of colitis via stimulation of IL-22 production by innate lymphoid cells (ILCs) (53). These cells, present in the intestinal tract, have also been shown to limit $\mathrm{T}$ cell responses through antigen presentation, possibly by outcompeting dendritic cells (54). In addition to IL-17, IL-23 has serious implications in the progression of colitis, being a key cytokine in pathogenic Th17 differentiation. Gut epithelium suppresses IL-23 via IL-25 production, thereby limiting the expansion of highly pathogenic Th17 cells in the intestine (55). Therefore, the intestinal immune system has regulatory mechanisms in place to protect against inflammation.

Currently, few treatments are available for MS, and a major interest exists for treatments which block infiltration of T cells into the CNS and manipulate T helper cell plasticity (reviewed in ref. 56). Our study highlights a new therapeutic strategy for MS, which increases $\mathrm{T}$ helper cell plasticity and promotes $\mathrm{T}$ cell trafficking away from the CNS, thus providing protection against the development of the disease.

\footnotetext{
1. Szabo SJ, Kim ST, Costa GL, Zhang X, Fathman CG, Glimcher LH. A novel transcription factor, T-bet, directs Th1 lineage commitment. Cell. 2000;100(6):655-669.

2. Zheng W, Flavell RA. The transcription factor GATA-3 is necessary and sufficient for Th2 cytokine gene expression in CD4 T cells. Cell.
}

\section{Methods}

Mice. Bcl11 $b^{F / F} / \mathrm{dLck}$-iCre mice were previously described (27). OT2 transgenic, CD45.1/Rag2-/, and 2D2 transgenic mice were from Jackson Laboratories. All mice were housed in pathogen-free conditions in the Albany Medical Center Animal Facility. All experiments were conducted on 8- to 16-week-old mice. Mice were paired with age-matched controls.

EAE induction. Emulsions containing $4 \mathrm{mg} / \mathrm{ml}$ of mycobacterium tuberculosis in Incomplete Freund's Adjuvant (Sigma-Aldrich) and $2 \mathrm{mg} / \mathrm{ml}$ MOG $_{35-55}$ peptide were injected subcutaneously above each hind flank of the 8- to 10-week-old female mice. 200 ng pertussis toxin (List Biological Inc.) were injected i.p. on day 0 and day 1 . Beginning with day 7 , disease progression was monitored. Clinical scoring was established as follows: score 1: flaccid tail; score 2: weak hind limbs; score 3: hind limb paralysis; score 4: quadriplegia.

VAD deficiency. Pregnant females were placed on a VAD (0 IU/g; LabDiet) diet between days 16 and 20 of pregnancy. Mice remained on a vitamin Adeficient diet for the remainder of experiments.

Treatment with anti-IL-4- or anti- $\alpha_{4} \beta_{7}$-neutralizing antibodies or IL-4 cytokine. $B c l 11 b^{F / F} / \mathrm{dLckd}$-iCre and WT mice were treated with either $100 \mu \mathrm{g}$ of antiLPAM-1/ $\alpha_{4} \beta_{7}$-neutralizing (DATK32) antibodies or $100 \mu$ g anti-IL-4 (11B11) or IgG (BioXcell) by i.p. injections starting 1 day prior to immunization. Every third day, mice were treated with $50 \mu \mathrm{g}$ of the same neutralizing antibody for the remainder of the experiment. $1 \mu \mathrm{g}$ recombinant IL-4 (Peprotech) or saline was administered on the same day as EAE induction via i.p. injection, and mice were further treated every third day for the remainder of the experiment. $M O G /$ alum immunization. Seven days after EAE induction, mice were immunized by i.p. administration of $\mathrm{MOG}_{35-55}$ in alum $(2 \mathrm{mg} / \mathrm{ml})$ or alum alone. Mice injected with $\mathrm{MOG}_{35-55}$ in alum also received two $50 \mu \mathrm{g}$ i.p. injections of Pam3CSK4 (Invivogen).

Statistics. Differences between groups were determined by a 2-tailed Student's $t$ test (unequal variance). $P \leq 0.05$ was considered significant. All values were expressed as mean \pm SEM.

Study approval. All mouse procedures were approved by the Albany Medical Center Institutional Animal Care and Use Committee.

Complete methods can be found in Supplemental Methods.

\section{Acknowledgments}

We greatly acknowledge Krupa Pandey, James Wymer, Allen Gerber, Michelle Lennartz, Jon Harton, Kate MacNamara (Albany Medical Center), and Diana Albu (Eisai) for valuable suggestions and exciting discussions. Special thanks are given to Neal G. Copeland, Nancy A. Jenkins (MHRI), and Pentao Liu (Sanger Institute) for mice. We greatly acknowledge Adrian Avram for graphical presentation and Douglas Cohn, Victoria Boppert and Hattie Wang for care of the mice. Grant support was from NIH grants AI067846 and AI078273 (to D. Avram).

Received for publication March 25, 2013, and accepted in revised form October 3, 2013.

Address correspondence to: Dorina Avram, Albany Medical College, 47 New Scotland Ave., Albany, New York 12208 MS349, MC151, USA. Phone: 518.262.6731; Fax: 518.262.5669; E-mail: avramd@mail.amc.edu.

1997;89(4):587-596.

3. O'Shea JJ, Paul WE. Mechanisms underlying lineage commitment and plasticity of helper CD4+ T cells. Science. 2010;327(5969):1098-1102.

4. Ivanov II, et al. The orphan nuclear receptor RORgammat directs the differentiation program of proinflammatory IL-17+ T helper cells. Cell.
2006;126(6):1121-1133

5. Codarri L, et al. RORgammat drives production of the cytokine GM-CSF in helper T cells, which is essential for the effector phase of autoimmune neuroinflammation. Nat Immunol. 2011;12(6):560-567.

6. El-Behi M, et al. The encephalitogenicity of $\mathrm{T}(\mathrm{H}) 17$ cells is dependent on IL-1- and IL-23-induced pro- 
duction of the cytokine GM-CSF. Nat Immunol. 2011;12(6):568-575.

7. Crotty S. Follicular helper CD4 T cells (TFH). Annu Rev Immunol. 2011;29:621-663.

8. Vinuesa CG, Cyster JG. How T cells earn the follicular rite of passage. Immunity. 2011;35(5):671-680.

9. Harrington LE, et al. Interleukin 17-producing $\mathrm{CD} 4+$ effector $\mathrm{T}$ cells develop via a lineage distinct from the Thelper type 1 and 2 lineages. Nat Immunol. 2005;6(11):1123-1132.

10. Zhou L, Chong MM, Littman DR. Plasticity of CD4+ T cell lineage differentiation. Immunity. 2009;30(5):646-655.

11. Nakayamada S, Takahashi H, Kanno Y, O'Shea JJ. Helper T cell diversity and plasticity. Curr Opin Immunol. 2012;24(3):297-302.

12. Lee $Y K$, et al. Late developmental plasticity in the $T$ helper 17 lineage. Immunity. 2009;30(1):92-107.

13. Hirota K, et al. Fate mapping of IL-17-producing $\mathrm{T}$ cells in inflammatory responses. Nat Immunol. 2011;12(3):255-263.

14. Hegazy AN, et al. Interferons direct Th2 cell reprogramming to generate a stable GATA-3(+)T-bet $(+)$ cell subset with combined Th2 and Th1 cell functions. Immunity. 2010;32(1):116-128.

15. Zaretsky AG, Taylor JJ, King IL, Marshall FA, Mohrs $\mathrm{M}$, Pearce EJ. T follicular helper cells differentiate from Th2 cells in response to helminth antigens. J Exp Med. 2009;206(5):991-999.

16. Lu KT, et al. Functional and epigenetic studies reveal multistep differentiation and plasticity of in vitro-generated and in vivo-derived follicular $\mathrm{T}$ helper cells. Immunity. 2011;35(4):622-632.

17. Avram D, Fields A, Pretty On Top K, Nevrivy DJ, Ishmael JE, Leid M. Isolation of a novel family of $\mathrm{C}(2) \mathrm{H}(2)$ zinc finger proteins implicated in transcriptional repression mediated by chicken ovalbumin upstream promoter transcription factor (COUP-TF) orphan nuclear receptors. J Biol Chem. 2000;275(14):10315-10322.

18. Avram D, Fields A, Senawong T, Topark-Ngarm A, Leid M. COUP-TF (chicken ovalbumin upstream promoter transcription factor)-interacting protein 1 (CTIP1) is a sequence-specific DNA binding protein. Biochem J. 2002;368(pt 2):555-563.

19. Cismasiu VB, Adamo K, Gecewicz J, Duque J, Lin Q, Avram D. BCL11B functionally associates with the NuRD complex in T lymphocytes to repress targeted promoter. Oncogene. 2005;24(45):6753-6764

20. Cismasiu VB, et al. BCL11B participates in the activation of IL2 gene expression in CD4+ T lymphocytes. Blood. 2006;108(8):2695-2702.

21. Albu DI, et al. BCL11B is required for positive selection and survival of double-positive thymocytes. JExp Med. 2007;204(12):3003-3015.

22. Wakabayashi Y, et al. Bcl11b is required for differentiation and survival of alphabeta $\mathrm{T}$ lymphocytes. Nat Immunol. 2003;4(6):533-539.

23. Ikawa $T$, et al. An essential developmental checkpoint for production of the $\mathrm{T}$ cell lineage. Science. 2010;329(5987):93-96.

24. Li L, Leid M, Rothenberg EV. An early T cell lineage commitment checkpoint dependent on the transcrip- tion factor Bcl11b. Science. 2010;329(5987):89-93.

25. Li P, et al. Reprogramming of $\mathrm{T}$ cells to natural killer-like cells upon $B c l 11 b$ deletion. Science. 2010;329(5987):85-89.

26. Albu DI, et al. Transcription factor Bcl11b controls selection of invariant natural killer T-cells by regulating glycolipid presentation in doublepositive thymocytes. Proc Natl Acad Sci U S A. 2011; 108(15):6211-6216

27. Zhang S, et al. Antigen-specific clonal expansion and cytolytic effector function of CD8+ T lymphocytes depend on the transcription factor Bcl11b. J Exp Med. 2010;207(8):1687-1699.

28. Vanvalkenburgh J, et al. Critical role of $B c l 11 b$ in suppressor function of $\mathrm{T}$ regulatory cells and prevention of inflammatory bowel disease. J Exp Med. 2011;208(10):2069-2081.

29. Asnagli H, Afkarian M, Murphy KM. Cutting edge: Identification of an alternative GATA-3 promoter directing tissue-specific gene expression in mouse and human. J Immunol. 2002;168(9):4268-4271.

30. Bettelli E, Pagany M, Weiner HL, Linington C, Sobel RA, Kuchroo VK. Myelin oligodendrocyte glycoprotein-specific $\mathrm{T}$ cell receptor transgenic mice develop spontaneous autoimmune optic neuritis. J Exp Med. 2003;197(9):1073-1081.

31. Reboldi A, et al. C-C chemokine receptor 6-regulated entry of TH-17 cells into the CNS through the choroid plexus is required for the initiation of EAE. Nat Immunol. 2009;10(5):514-523.

32. Bauer $\mathrm{M}$, et al. Beta1 integrins differentially control extravasation of inflammatory cell subsets into the CNS during autoimmunity. Proc Natl Acad Sci US A. 2009;106(6):1920-1925

33. Shulman Z, et al. Lymphocyte crawling and transendothelial migration require chemokine triggering of high-affinity LFA-1 integrin. Immunity. 2009;30(3):384-396.

34. Berlin C, et al. Alpha 4 beta 7 integrin mediates lymphocyte binding to the mucosal vascular addressin MAdCAM-1. Cell. 1993;74(1):185-195.

35. Kunkel EJ, et al. Lymphocyte CC chemokine receptor 9 and epithelial thymus-expressed chemokine (TECK) expression distinguish the small intestinal immune compartment: Epithelial expression of tissue-specific chemokines as an organizing principle in regional immunity. J Exp Med. 2000;192(5):761-768.

36. Stenstad H, et al. Gut-associated lymphoid tissueprimed CD4+ T cells display CCR9-dependent and -independent homing to the small intestine. Blood. 2006;107(9):3447-3454

37. Yokota A, et al. GM-CSF and IL-4 synergistically trigger dendritic cells to acquire retinoic acid-producing capacity. Int Immunol. 2009;21(4):361-377.

38. Iwata M, Hirakiyama A, Eshima Y, Kagechika H, Kato C, Song SY. Retinoic acid imprints gut-homing specificity on T cells. Immunity. 2004;21(4):527-538.

39. Manicassamy S, Pulendran B. Retinoic aciddependent regulation of immune responses by dendritic cells and macrophages. Semin Immunol. 2009;21(1):22-27.

40. Guilliams $M$, et al. Skin-draining lymph nodes contain dermis-derived CD103(-) dendritic cells that constitutively produce retinoic acid and induce Foxp3(+) regulatory T cells. Blood. 2010;115(10):1958-1968.

41. Suzuki K, et al. The sensing of environmental stimuli by follicular dendritic cells promotes immunoglobulin A generation in the gut. Immunity. 2010;33(1):71-83.

42. Wilson CB, Rowell E, Sekimata M. Epigenetic control of T-helper-cell differentiation. Nat Rev Immunol. 2009;9(2):91-105

43. Furlan R, et al. Central nervous system gene therapy with interleukin-4 inhibits progression of ongoing relapsing-remitting autoimmune encephalomyelitis in Biozzi AB/H mice. Gene Ther. 2001;8(1):13-19.

44. Massacesi L, Abbamondi AL, Giorgi C, Sarlo F, Lolli F, Amaducci L. Suppression of experimental allergic encephalomyelitis by retinoic acid. J Neurol Sci. 1987;80(1):55-64.

45. Xiao S, et al. Retinoic acid increases Foxp3+ regulatory $\mathrm{T}$ cells and inhibits development of Th17 cells by enhancing TGF-beta-driven Smad3 signaling and inhibiting IL-6 and IL-23 receptor expression. J Immunol. 2008;181(4):2277-2284.

46. Takahashi $\mathrm{H}$, et al. TGF-beta and retinoic acid induce the microRNA miR-10a, which targets Bcl-6 and constrains the plasticity of helper T cells. Nat Immunol. 2012;13(6):587-595.

47. Hall JA, Grainger JR, Spencer SP, Belkaid Y. The role of retinoic acid in tolerance and immunity. Immunity. 2011;35(1):13-22.

48. Ericsson A, Svensson M, Arya A, Agace WW. CCL25/CCR9 promotes the induction and function of CD103 on intestinal intraepithelial lymphocytes. Eur J Immunol. 2004;34(10):2720-2729.

49. Wang C, Kang SG, HogenEsch H, Love PE, Kim $\mathrm{CH}$. Retinoic acid determines the precise tissue tropism of inflammatory Th17 cells in the intestine. J Immunol. 2010;184(10):5519-5526.

50. Esplugues E, et al. Control of TH17 cells occurs in the small intestine. Nature. 2011;475(7357):514-518.

51 . O'Connor W, et al. A protective function for interleukin $17 \mathrm{~A}$ in $\mathrm{T}$ cell-mediated intestinal inflammation. Nat Immunol. 2009;10(6):603-609.

52 . Hueber W, et al. Secukinumab, a human anti-IL$17 \mathrm{~A}$ monoclonal antibody, for moderate to severe Crohn's disease: unexpected results of a randomised, double-blind placebo-controlled trial. Gut. 2012;61(12):1693-1700.

53. Mielke LA, et al. Retinoic acid expression associates with enhanced IL-22 production by gammadelta $\mathrm{T}$ cells and innate lymphoid cells and attenuation of intestinal inflammation. J Exp Med. 2013;210(6):1117-1124.

54. Hepworth MR, et al. Innate lymphoid cells regulate CD4+ T-cell responses to intestinal commensal bacteria. Nature. 2013;498(7452):113-117.

55. Zaph C, et al. Commensal-dependent expression of IL-25 regulates the IL-23-IL-17 axis in the intestine. J Exp Med. 2008;205(10):2191-2198.

56 . Becher B, Segal BM. T(H)17 cytokines in autoimmune neuro-inflammation. Curr Opin Immunol. 2011;23(6):707-712. 